Document downloaded from:

http://hdl.handle.net/10251/125095

This paper must be cited as:

Giner-Sanz, JJ.; Ortega Navarro, EM.; Pérez-Herranz, V. (2018). Optimization of the Perturbation Amplitude for EIS Measurements Using a Total Harmonic Distortion Based Method. Journal of The Electrochemical Society. 165(10):E488-E497.

https://doi.org/10.1149/2.1021810jes

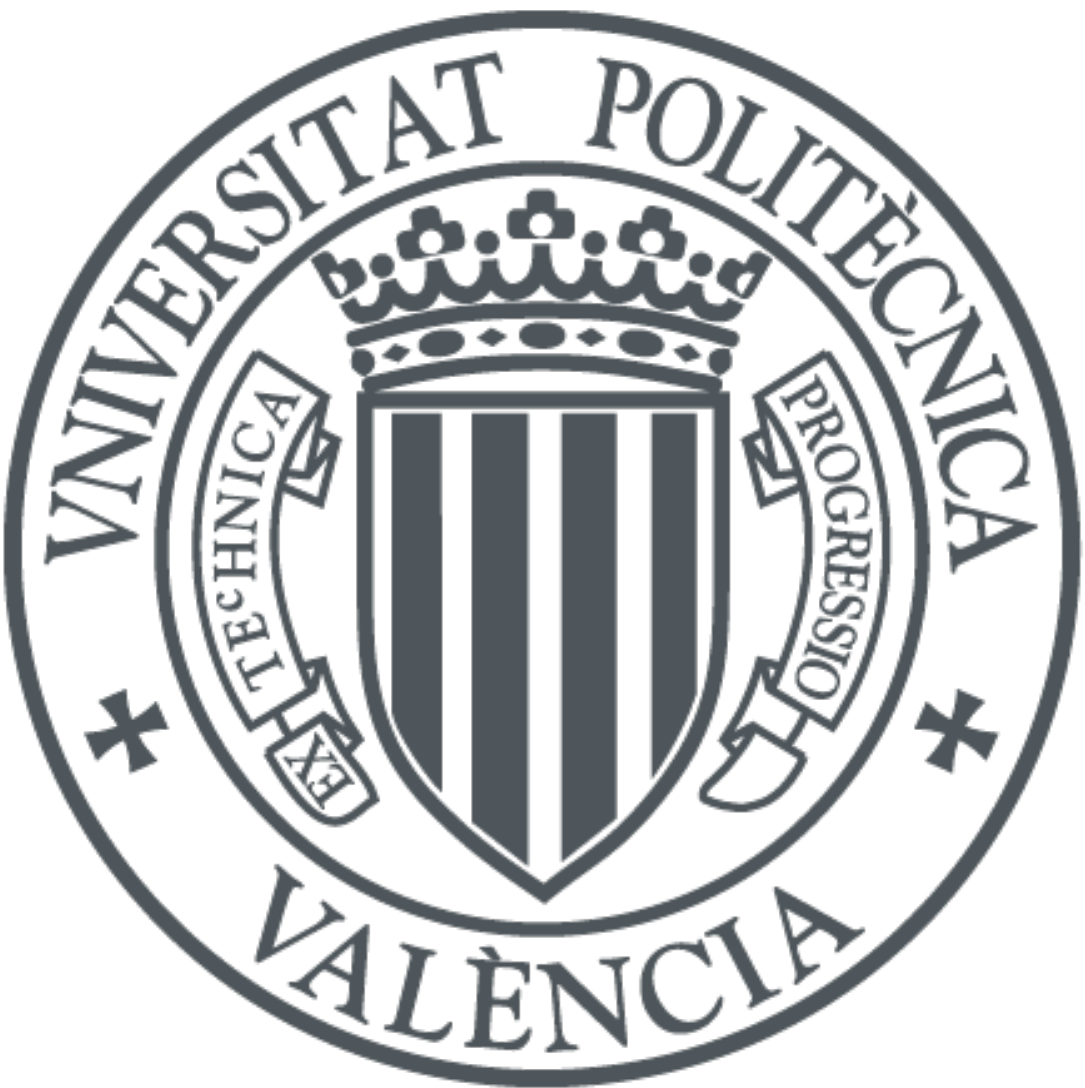

The final publication is available at

http://doi.org/10.1149/2.1021810jes

Copyright The Electrochemical Society

Additional Information 


\title{
Optimization of the perturbation amplitude for EIS measurements using a Total Harmonic Distortion based method
}

\author{
J. J. Giner-Sanz, E. M. Ortega, V. Pérez-Herranz* \\ IEC group, Depto. Ingeniería Química y Nuclear, Universitat Politècnica de València \\ Camino de Vera S/N, 46022 Valencia, Spain \\ *Corresponding author. Tel.: +34-96-3877632; fax: +34-96-3877639; \\ E-mail address: vperez@iqn.upv.es (V.Pérez-Herranz)
}

\section{Abstract:}

Ohm's generalized law defines the concept of impedance. This law, and thus the definition itself, are only valid if the system fulfills the linearity condition. However, electrochemical systems are typically highly nonlinear. Consequently, the linearity condition can only be achieved in these systems if a low perturbation amplitude is used for performing EIS measurements. Nevertheless, the use of low amplitude perturbations leads to low signal-to-noise ratios, which result in high measurement errors. The concept of optimum amplitude arises from this tradeoff: the perturbation has to have an amplitude big enough in order to minimize the measurement errors (i.e. maximize the SNR), but at the same time, the perturbation has to have an amplitude small enough to avoid the generation of significant nonlinear effects that would distort the measured EIS spectra. In a previous work, a linearity assessment quantitative method based on the total harmonic distortion parameter was developed. In this work, the aforementioned THD method was applied for the perturbation amplitude selection for EIS measurements in a highly nonlinear model system: the cathodic electrode of an alkaline water electrolyser. The THD method successfully obtained the optimum amplitudes both, for a constant amplitude strategy and for a frequency dependent strategy. The THD method also allowed to obtain the noise structure and to quantify the nonlinear effects. This method is slightly superior to the $\wp U$ method, a method based on the harmonic analysis of the output signal that was developed in earlier works.

Keywords: Total harmonic distortion, Electrochemical impedance spectroscopy, Frequency dependent perturbation amplitude, Noise structure, Amplitude optimization. 


\section{Introduction}

Impedance spectroscopy (IS) is a group of non-destructive analytical methods that can be divided in two subcategories: dielectric IS, and electrochemical IS (EIS) [1]. On the one hand, as its name suggests, dielectric IS involves the study of dielectric materials, in which electronic conduction dominates. On the other hand, EIS deals with materials in which ionic conductivity dominates over electronic conductivity [2]. To date, this technique has been applied in a wide range of applications, such as supercapacitors [35], corrosion [6-11], solar cells [12-14], electrolysers [15-18], electrochemical sensors [19-21], batteries [22-25], and fuel cells [26-29], amongst others. Today, it is considered as one of the fundamental electrochemical techniques [30].

This electrochemical method is based on the application of sinusoidal signals (AC component) of different frequencies, superimposed to a constant signal (DC component); and the analysis of the response of the system under study. In general, the frequency spans over a wide range that usually extends from some $\mathrm{MHz}$ down to few $\mathrm{mHz}$ [31]. In the case of potentiostatic EIS, the perturbation is a voltage signal, while the output is a current signal; whereas, in galvanostatic EIS, the perturbation is a current signal and the output is a voltage signal [32]. EIS measurements can be performed both, in 2- or 3- electrode configuration, depending on the presence (3electrode configuration) or the absence (2-electrode configuration) of a constant and known potential electrode (i.e. reference electrode) in the system [1]. In some particular systems (v.g. ion-exchange membrane systems) a 4-electrode configuration is used [33].

This technique requires the system to behave pseudo-linearly, since the definition of impedance (i.e. complex Ohm's law) is only valid for linear systems [34]. Electrochemical systems are intrinsically nonlinear systems since they present nonlinear effects, as nonlinear kinetics (Butler-Volmer's kinetics) and saturation [35, 36]. Consequently, pseudo-linear behavior can only be achieved in electrochemical systems if a low perturbation amplitude is applied (i.e. small signal approximation) [37]. However, the use of small perturbation amplitudes leads to small signal-to-noise ratios (SNR) [38], which has a negative impact on the quality of the measured spectra. So, even if theoretically an infinitely small perturbation amplitude $(\rightarrow 0)$ is required for the electrochemical system to behave pseudo-linearly [39]; in practice, the amplitude of the AC signal has to be chosen in order to be small enough to guarantee the pseudolinear behavior of the system, but large enough to be able to measure the response of the system [1]. In this context, the concept of optimum perturbation amplitude arises: the optimum amplitude corresponds with the amplitude that maximizes the SNR without violating significantly the linearity condition [40]. At the end of the day, the 
optimum perturbation amplitude is defined by the trade-off between the maximization of the SNR, and the fulfilment of the linearity behavior condition.

Since the selection of the perturbation amplitude is critical for the quality of the measured EIS spectra, it is a fundamental part of the experimental design of any experiment that involves EIS [41]. The optimum perturbation amplitude depends strongly on the studied electrochemical system [30], and can even vary from one operation point to another for a given system [40]. However, for potentiostatic EIS, there is a well-stablished criterion according to which the applied perturbation amplitude should be lower than the thermal voltage $\left(V_{\text {termal }}\right)$ [32], given by:

$$
V_{\text {termal }}=\frac{k \cdot T}{e}
$$

Where $k \approx 1.38 \times 10^{-23} \mathrm{~J} \cdot K^{-1}$ denotes the Boltzmann constant; $e \approx 1.60 \times$ $10^{-19} \mathrm{C}$ represents the elementary charge; and $T$ is the temperature, expressed in $K$. At room temperature $(298 \mathrm{~K})$, the thermal voltage is around $25 \mathrm{mV}$. This result leads to the generally accepted guideline of using voltage perturbations of a few tenths of $\mathrm{mVs}$ for performing potentiostatic EIS measurements. Unfortunately, this guideline is hardly transposable to galvanostatic EIS.

In their book [30], Orazem and Tribollet, present a method that allows to estimate theoretically the maximum perturbation amplitude that guarantees the fulfilment of the linearity condition. The main limitation of this method is that it requires a fair amount of knowledge of the studied system, such as a model of the system and its parameters. However, in general, these data are not available when studying a new system; and consequently, the method cannot be used for the amplitude selection in the experimental design phase.

A common practice for amplitude selection is to repeat the EIS measurement for different perturbation amplitudes, and select the maximum amplitude that does not distort significantly the obtained spectra [1]. This strategy was used for example by Gode's team [42], by Yuan and co-workers [43], and by Fernández-Pulido and coworkers [44]. However, this selection methodology presents several important drawbacks. First, the EIS spectra may change due to other causes than the perturbation amplitude (v.g. time drift); and therefore, when using this method, a suboptimal amplitude may be selected because a variation in the spectra was observed, but this variation was not due to the effect of $A C$ amplitude. Second, the term "distort significantly" is a fairly fuzzy concept. The criteria to identify a significant distortion in EIS spectra are quite indeterminate, and the identification generally depends strongly on the subjectivity of the analyst. Finally, this selection method can 
only be used for the traditional strategy of using a constant perturbation amplitude for all the frequencies considered in the frequency sweep; but it is not suitable for a variable amplitude strategy, in which a different amplitude is applied at each frequency. In order to overcome these limitations, it is advisable to use a systematic and subjectivity-free method for selecting the perturbation amplitude.

Since the optimum amplitude is determined by the linearity of the system, linearity assessment methods are very good candidates for the selection of the perturbation amplitude. A large number of such methods can be found in bibliography [45]. They can be grouped in 3 main categories: Experimental linearity assessment methods, linearity assessment methods based on Kramers-Kronig Transforms (KKT), and linearity assessment methods based on Harmonic Analysis (HA) [31].

First, the experimental linearity assessment methods group encompasses AC plots, resolution plots and Lissajous plots. All these plots allow assessing linearity in real time during EIS measurements, by monitoring directly the perturbation and output signals in the time domain [30]. A great number of today's commercial softwares for EIS measurements display these plots during data acquisition. They present two major limitations. On the one hand, these methods are qualitative methods that can detect nonlinearities, but that cannot quantify them. On the other hand, unlike severe nonlinearities, which are easily identified in these plots; low and moderate ones are not so clear, and their identification is sometimes ambiguous [40]. For these reasons, the use of experimental linearity assessment methods should be reserved for auxiliary experimental validation of EIS measurements, and they should not be used for rigorous selection of EIS perturbation amplitudes.

Second, as their name suggests, the KKT based linearity assessment methods are linearity assessment methods based on the integral Kramers-Kronig relations which have been proven to be very powerful validation tools for EIS spectra [46]. A thorough review of the KKT methods available in literature for EIS spectra validation was presented by Agarwal and Orazem [47]. Voigt method developed by Boukamp and coworkers $[48,49]$, and the measurement model method developed by Orazem's group $[47,50-58]$ are the main examples of validation methods based on KKT. It has been proven that KKT are very highly insensitive to linearity violation [59-61]. This makes KKT based linearity assessment methods unsuitable for the selection of perturbation amplitudes.

Finally, the HA linearity assessment methods are based on the analysis of the system's output in the frequency domain. These methods take advantage of the well-known property of nonlinear systems according to which the response of a nonlinear system to a mono-frequency sinusoidal perturbation is a superposition of different sine waves 
of different frequencies (i.e. fundamental component and non-fundamental harmonics) [62]. The generation of non-fundamental harmonics due to nonlinearity has been widely demonstrated in literature. Diard's team work [63-70], Darowicki's team work [71-74], and Van Gheem's team work [75, 76] are some of the aforementioned studies. The HA methods assess linearity by quantifying the nonfundamental harmonic content in the output signal. Several examples of HA linearity assessment methods are available in bibliography, such as the method presented by Pintelon's team [77, 78], Popkirov and Schindler's method [79, 80], the total distortion based method [81], and the method developed in previous works [39, 40]. The HA methods have two great strong points. On the one hand, they are quantitative methods that allow quantifying (i.e. in an objective manner) the level of nonlinearity. On the other hand, they are highly sensitive to nonlinearities: in fact it has been shown that they are more sensitive to nonlinearities than the EIS spectra themselves $[18,39]$. These advantages make them very good candidates for being used, in the EIS context, for the amplitude selection.

This work's goal is to present a methodology for EIS perturbation amplitude selection, and illustrate it by applying it to a highly nonlinear electrochemical system, the cathodic electrode of an alkaline hydrogen evolution cell. The presented methodology is based on the total harmonic distortion based method described in a previous work [81]. The results obtained in this work using the total harmonic distortion based method, will be compared with the results obtained in a previous work [40] using another HA method. As in the aforementioned work, two strategies were considered in the present work: the conventional constant amplitude strategy, and the frequency dependent amplitude strategy. 


\section{Total harmonic distortion method}

The total harmonic distortion method was presented in detail in a previous work [81]. Its basis is the use of a quantitative indicator (i.e. Total Harmonic Distortion, THD) of the non-fundamental harmonic content in the output signal. The THD is defined as [82]:

$$
\mathcal{J H D} U=\frac{1}{|\widehat{U}|_{1}} \cdot \sqrt{\sum_{i=2}^{+\infty}|\widehat{U}|_{i}^{2}}
$$

Where $|\widehat{U}|$ denotes the amplitude of the Fourier transform of the voltage signal (i.e. output signal in the frequency domain). Subscript 1 corresponds to the fundamental component; whereas subscripts $\geq 2$ are associated to non-fundamental harmonics. In general, THD values are expressed as a percentage of the fundamental component. This parameter has been used in EIS context in several works [83-86].

Figure 1 of reference [81] outlines the total harmonic distortion method. It consists in three main steps. In the first one, the EIS spectrum is measured as usual, storing as well the raw signals in the time domain, $I(t)$ and $U(t)$, for each excited frequency. In the second step, the time domain signals are transformed to the frequency domain, $\hat{I}(\vartheta)$ and $\widehat{U}(\vartheta)$, using a Fast Fourier Transform (FFT) algorithm. In the third step, the THD is calculated using expression (2), for each excited frequency. In this way, the THD value (i.e. non-fundamental harmonic content) is obtained for each excited frequency (i.e. frequency at which the impedance was measured). On the one hand, the THD plot is obtained by representing $\mathcal{T H} \mathcal{H} \mathcal{D} U$ versus the excited frequency. On the other hand, the critical parameter, $\mathcal{T H} \mathcal{H} U_{c}$, is calculated using the following expression:

$$
\mathcal{T H} \mathcal{H} \mathcal{D} U_{c}=\max _{k \in\left\{1 ; \cdots ; N_{f}\right\}} \mathcal{T H} \mathcal{H} U_{k}
$$

Where $N_{f}$ stands for the number of frequencies at which the impedance was measured, and $\mathcal{T H} \mathcal{H} U_{k}$ denotes the $\mathcal{T} \mathcal{H} \mathcal{D} U$ for the $k$-th excited frequency. The excited frequency at which the non-fundamental harmonic content is maximum (i.e. frequency at which $\mathcal{T H} \mathcal{H} U_{k}=\mathcal{T} \mathcal{H} \mathcal{D} U_{c}$ ) is defined as the critical frequency, $f_{c}$.

In this work, the total harmonic distortion method was implemented in Labview $\circledR$. The program takes as input the NOVA®'s output, which is a .txt file that contains the current and voltage signals in the time domain and in the frequency domain. 
The aforementioned analysis was repeated for different perturbation amplitudes. As it was thoroughly explained in reference [81], the trend of parameter $\mathcal{T H} \mathcal{H} U$ with the perturbation amplitude, $\Delta I$, allows to distinguish the linear behavior zone from the nonlinear behavior zone. For low amplitudes, an increase in $\Delta I$ causes a decrease in $\mathcal{T H} \mathcal{H} U$. In this zone, the improvement of the SNR dominates over the nonlinear effects: the system behaves pseudo-linearly (i.e. linear behavior zone). On the contrary, for high amplitudes, an increase in $\Delta I$ causes an increase in $\mathcal{T H} \mathcal{H} U$. In this zone, the nonlinear effects dominate over the improvement of the SNR: the system behaves clearly as a nonlinear system (i.e. nonlinear behavior zone). Moreover, parameter $\mathcal{T H} \mathcal{H} U$ can be decomposed in two contributions: the first one associated to the noise, and the second one associated to nonlinear effects [81]. Thus, for the $k$ th excited frequency:

$$
\mathcal{T H} \mathcal{H} U_{k}(\Delta I)=\mathcal{T} \mathcal{H} \mathcal{D} U_{k}^{\text {noise }}(\Delta I)+\mathcal{T} \mathcal{H} \mathcal{D} U_{k}^{\text {nonlinear }}(\Delta I)
$$

Where $\mathcal{T H} \mathcal{H} U_{k}^{\text {noise }}$ stands for the noise related component of parameter $\mathcal{T H} \mathcal{H} U$ for the $k$-th excited frequency; and $\mathcal{T} \mathcal{H} \mathcal{D} U_{k}^{\text {nonlinear }}$ denotes the component of parameter $\mathcal{T H} \mathcal{H} U$ related to nonlinear effects, for the $k$-th excited frequency. The noise related component decreases with the perturbation amplitude according to the following expression [81]:

$$
\mathcal{T H} \mathcal{H} U_{k}^{\text {noise }}(\Delta I)=\frac{\lambda_{k}}{\Delta I}
$$

Where $\lambda_{k}$ stands for the effective noise parameter for the $k$-th excited frequency. This parameter has current dimensions, and can be obtained from fitting the linear behavior zone of the experimental $\mathcal{T H} \mathcal{H} U$ curve. Parameter $\lambda_{k}$ is defined as:

$$
\lambda_{k}=\frac{\chi_{k}}{|\mathrm{Z}|_{k}}
$$

Where $|\mathrm{Z}|_{k}$ denotes the impedance modulus for the $k$-th excited frequency; and $\chi_{k}$ stands for the total noise parameter for the $k$-th excited frequency. It has voltage dimensions, and is defined as:

$$
\chi_{k}=\sqrt{\sum_{i=2}^{+\infty}\left(\Delta U_{k, i}^{\text {noise }}\right)^{2}}
$$


In the above expression, $\Delta U_{k, i}^{\text {noise }}$ is the $i$-th component of the output signal associated to noise, for the $k$-th excited frequency. Parameter $\chi_{k}$ quantifies the total noise level for the $k$-th frequency measurement; whereas $\lambda_{k}$ quantifies the effective noise level for the $k$-th frequency measurement.

Once $\mathcal{T} \mathcal{H} \mathcal{D} U_{k}^{\text {noise }}$ is known, using equation 4, the component of $\mathcal{T} \mathcal{H} \mathcal{D} U$ related to nonlinear effects can be obtained by subtraction:

$$
\mathcal{T} \mathcal{H} \mathcal{D} U_{k}^{\text {nonlinear }}(\Delta I)=\mathcal{T} \mathcal{H} \mathcal{D} U_{k}(\Delta I)-\mathcal{T} \mathcal{H} \mathcal{D} U_{k}^{\text {noise }}(\Delta I)
$$

The great advantage of the total harmonic distortion method, over the $\wp U$ method $[39,40]$, is that THD contains simultaneously information on noise and nonlinearity; while parameter $\wp U$ only contains information on noise in the linear behavior zone, and information on nonlinearity in the nonlinear behavior zone. 


\section{Amplitude selection methodology}

In this work, two different EIS measurement strategies were considered: On one side, the traditional constant amplitude strategy; and on the other side, a frequency dependent amplitude strategy. As explained in section 1, from a practical point of view, the optimum perturbation amplitude is the maximum amplitude for which nonlinear effects are negligible in comparison with the noise. This argument can be applied for the most unfavorable excited frequency, defining a single amplitude for all the frequencies (i.e. constant amplitude strategy); or it can be applied separately to each excited frequency, selecting a perturbation amplitude for each one of them (i.e. frequency dependent amplitude strategy).

In order to fulfill this work's aim, the galvanostatic EIS spectrum of the system was measured using different perturbation amplitudes. Then, the total harmonic distortion method described in section 2, was applied to each one of the experimental EIS spectra, obtaining the THD plot and the critical parameter for each perturbation amplitude.

On the one hand, for the constant amplitude strategy, the critical parameter curve was used for selecting the perturbation amplitude. This curve is built by representing the critical parameter, $\mathcal{T} \mathcal{H} \mathcal{D} U_{c}$, versus $\Delta I$. The optimum amplitude corresponds with the perturbation amplitude that minimizes the critical parameter (i.e. the abscissa of the minimum of the critical parameter curve) [40].

On the other hand, for the frequency dependent amplitude strategy, the individual frequency THD plots were used for selecting the perturbation amplitude for each excited frequency. These plots are obtained by representing parameter $\mathcal{T} \mathcal{H} \mathcal{D} U$ versus $\Delta I$, for each excited frequency. The optimum amplitude for a given excited frequency corresponds with the perturbation amplitude that minimizes the $\mathcal{T H} \mathcal{H} U$ parameter for that excited frequency (i.e. the abscissa of the minimum of its individual frequency THD plot) [40]. 


\section{$\underline{\text { 4. Experimental work }}$}

A highly nonlinear electrochemical system was selected in this work in order to illustrate the perturbation amplitude selection methodology presented in this work: the cathodic electrode of an alkaline water electrolyser. The experimental setup used here has been thoroughly described in previous works [40, 81]. Figure 2 of reference [81] shows a detailed diagram. Its main element is a patented [87] 3-electrode electrochemical thermostatted cell. The working electrode (WE), placed horizontally in order to minimize the effects of the generated bubbles, was a $0.5 \mathrm{~cm}^{2}$ (geometric area) nickel electrode produced at very high current densities using the procedure described in reference [88]. The counter-electrode (CE) was a nickel foam with very high surface area (Incofoam ${ }^{\circledR} 0.17 \mathrm{~cm}$ thick and 50 pores per linear inch). A commercial $\mathrm{Ag} / \mathrm{AgCl}$ $(3 \mathrm{M} \mathrm{KCl}$ ) electrode was used as reference electrode (RE), and an oxygen-free $30 \mathrm{wt} . \%$ $\mathrm{KOH}$ solution was used as electrolyte. Before each experiment, a 30 minutes potentiostatic pre-treatment was done, at $-1.6 \mathrm{~V}$ vs. $\mathrm{Ag} / \mathrm{AgCl}$, in order to ensure the reproducibility of the results [39].

As described in section 3, in this study, the EIS spectrum of the system was measured using different perturbation amplitudes. In this case, 12 peak to peak amplitudes were considered: $0.1 \mathrm{~mA} ; 0.5 \mathrm{~mA} ; 1 \mathrm{~mA} ; 2 \mathrm{~mA} ; . . . ; 10 \mathrm{~mA}$. All the experiments were done in galvanostatic mode, at the same operation point: an operation temperature of $30^{\circ} \mathrm{C}$, and a DC current of $-10 \mathrm{~mA}$. The maximum amplitude was selected in order to guarantee that, even for the maximum amplitude, the WE has a cathodic behavior during the whole measurement.

An Autolab $尺 302 \mathrm{~N}$ potentiostat/galvanostat with FRA module, controlled using NOVA® software, was used here to measure the EIS spectra from $10 \mathrm{kHz}$ to $5 \mathrm{mHz}$, with 10 frequencies per decade. The same measurement parameters were used in all the EIS measurements done in this work. They were selected using the methodology presented in a previous work [89], and are listed in table 1 of reference [81].

Reproducibility was assessed by measuring each EIS spectrum in triplicate, using a random order strategy. This strategy consists in performing the different measurements in a random order, instead of using the traditional sequential order. Using this random order strategy, the factors amplitude and time were orthogonalized, allowing to distinguish if the observed trends are due to the effect of the amplitude, or to a time drift [40]. 


\section{$\underline{\text { 5. Experimental results }}$}

\subsection{THD curves}

Figure 1, reproduced from a previous work [81], presents the THD curves of the output signal, for the different perturbation amplitudes. The first observation that can be extracted from this figure, is that the overall shape of the THD curve changes completely from low perturbation amplitudes (Figure 1.a) to high perturbation amplitudes (Figure 1.b). On the one side, in the low amplitude case, the THD curves present very low values for high and low frequencies, and significantly higher values for the midrange frequencies $(1-100 \mathrm{~Hz})$. On the other side, in the high amplitude case, the THD curves display 3 well defined zones: for low frequencies, $\mathcal{T H} \mathcal{H} U$ is approximately constant with frequency; for intermediate frequencies, the THD curves are monotonically decreasing curves with an inflexion point; and finally, for high frequencies, $\mathcal{T H} \mathcal{H} U$ is a very low constant, except for some peaks at characteristic frequencies ( 50 and $25 \mathrm{~Hz}$ ).

As it was explained in a previous work [81], the evolution of $\mathcal{T H} \mathcal{H D} U$ with the perturbation amplitude is an indicator of whether the system behaves pseudo-linearly or not. As it can be observed in figure 1.a, for low perturbation amplitudes, an increase in $\Delta I$ causes a decrease of the $\mathcal{T H} \mathcal{H} \mathcal{D} U$ value for all the excited frequencies. This observation is an evidence that for low perturbation amplitudes, the nonlinear effects are negligible compared to the improvement of the SNR. In other words, in the low perturbation amplitude range, the system behaves pseudo-linearly for every excited frequency. On the contrary, as it can be seen in figure 1.b, for high perturbation amplitudes, an increase in $\Delta I$ causes an increase of the $\mathcal{T H} \mathcal{H} U$ value. This observation shows that for high perturbation amplitudes, the nonlinear effects are no longer negligible compared to the improvement of the SNR: the system does not behave pseudo-linearly anymore. However, a closer look to figure 1.b, shows that for high excited frequencies the trend of $\mathcal{T H} \mathcal{H} U$ with $\Delta I$ is the same as the low-amplitudetrend. Thus, two different trends can be identified for high perturbation amplitudes: for low excited frequencies, an increase in $\Delta I$ causes a shift of $\mathcal{T H} \mathcal{H} U$ to higher values; whereas, for high frequencies, an increase in $\Delta I$ causes a shift of $\mathcal{T H} \mathcal{H} U$ to lower values: for high perturbation amplitudes, the system behaves pseudo-linearly for high excited frequencies, and behaves nonlinearly for low excited frequencies. This observation indicates that the generation of nonlinear effects is frequency dependent: a given system, can behave pseudo-linearly in a frequency range and nonlinearly in another frequency range, for a given perturbation amplitude. This fact justifies the consideration of a frequency dependent amplitude strategy: in order to maximize the improvement of the SNR, large amplitudes may be applied in the frequency range in which the system behaves pseudo-linearly even for very high amplitudes; and low 
amplitudes must be applied in the frequency range in which the system presents significant nonlinear effects.

As presented in section 2, the THD curves contain information on both, noise and nonlinear effects. In the following sections, this information is going to be extracted from the THD curves presented in this section (figure 1).

\subsection{Noise structure}

Noise plays a very important role in perturbation amplitude selection, since it is one of the two involved relevant factors (i.e. noise and nonlinear effects). For this reason, noise characterization and quantification is a good start for amplitude selection. As discussed in section 2, two of the outputs of the total harmonic distortion method are noise quantifiers: the effective noise parameter, $\lambda$; and the total noise parameter, $\chi$. The later quantifies the total noise level at a given excited frequency. The effective effects of this total noise level on the measurement are modulated by the impedance of the system at that excited frequency [81]. Parameter $\lambda$ arises from this fact: it quantifies the noise effective effect on the measurement at a given excited frequency. As explained in section 2 , the value of parameter $\lambda$ for the $k$-th excited frequency can be obtained by fitting the pseudo-linear behavior zone of the individual frequency THD plot related to frequency $k$, to the linear behavior model given by expression (5) [81]. This methodology was used in this work in order to calculate the effective noise parameter for each frequency. The obtained results are shown in figure 2 (black dots).

The first observation that can be extracted from figure 2 is that the effective noise parameter is clearly frequency dependent: not all frequencies present the same level of effective noise. Three zones can be identified on the aforementioned figure. First, in zone $C$ (i.e. high frequencies), parameter $\lambda$ is nearly frequency-independent and presents very low values. This implies that noise has very little effective effect on the measurements at high frequencies in this system. Second, in zone A (i.e. low frequencies), the effective noise parameter is slightly higher than in zone $C$, but it is still reasonably low. Moreover, in zone $A, \lambda$ is frequency-dependent, since it presents a decreasing trend with frequency. Finally, in zone B (i.e. intermediate frequencies), parameter $\lambda$ presents substantially higher values than in the other two zones. Furthermore, the curve presents peaks at certain characteristic frequencies, which correspond to the frequency of the electric grid and its subharmonics (i.e. $1 / n$ times the fundamental frequency): $50 \mathrm{~Hz}(n=1), 25 \mathrm{~Hz}(n=2), 12.5 \mathrm{~Hz}(n=4), 5 \mathrm{~Hz}(n=$ $10)$ and $2.5 \mathrm{~Hz}(n=20)$. For these frequencies, the effective noise parameter presents a markedly higher value, which implies that the effective noise level is significantly higher for these frequencies. Higher amplitudes will be needed in order to make nonlinear effects overcome the noise for these frequencies. These peaks are 
associated with the electric coupling of the measurement system with the electric grid. Electric coupling has already been identified as a major noise source in EIS measurements [41]. In this system, the $\lambda$ curve presents its highest peak at $25 \mathrm{~Hz}$ : this is the frequency at which the effective noise is maximum.

According to equation (6), the total noise parameter can be calculated by multiplying frequency-by-frequency the effective noise parameter by the impedance modulus at that frequency. The impedance modulus graph (i.e. red triangles) is superimposed to the effective noise parameter graph in figure 2. Combining (i.e. multiplying point-bypoint) both curves represented in figure 2, figure 3 was obtained. It plots the total noise parameter at each excited frequency. This curve presents the same 3 zones that were identified on the effective noise parameter. On the one hand, in zone $C$ (i.e. high frequencies), parameter $\chi$ is nearly frequency-independent and presents very low values. Thus, the total noise level is very low in zone $C$. Therefore, at high frequencies, the effective noise level is low because so is the total noise level. On the other hand, in zone A (i.e. low frequencies), the total noise parameter presents a clear decreasing trend with the excited frequency. For very low frequencies (i.e. under $10 \mathrm{mHz}$ ), the total noise levels are significant, and exceed $5 \mathrm{mV}$ (i.e. more than $25 \%$ of the maximum $\chi$ ). However, in this zone, the impedance modulus is maximum; and therefore, the effective noise level is substantially lower: the impedance modulus of the system, which is high in this frequency zone, dampens the high total noise levels, and leads to lower effective noise levels. Finally, in zone B (i.e. intermediate frequencies), parameter $\chi$ presents the same peak pattern that was observed for parameter $\lambda$, with the difference that in $\chi^{\prime}$ 's case the height of the peaks is approximately constant: the total noise parameter is equal for the excited frequencies associated to the $n=2,4,10,20$ subharmonics of the grid's frequency. These frequencies present the maximum total noise level, $19.5 \mathrm{mV}$. The $\lambda$ peaks present different heights since the impedance modulus differs from one peak frequency to another. These observations imply that the electric coupling with the grid introduces the same level of total noise in every frequency at which it occurs (i.e. grid subharmonics); and that the impedance modulus of the system modulates this total noise level, leading to different effective noise levels.

The total noise parameter definition (i.e. expression (7)) can be rewritten by breaking down the sum:

$$
\chi_{k}=\sqrt{\left(\Delta U_{k, \text { max }}^{\text {noise }}\right)^{2}+\sum_{\substack{i=2 \\ i \neq \max }}^{+\infty}\left(\Delta U_{k, i}^{\text {noise }}\right)^{2}}
$$


Where $\Delta U_{k, \text { max }}^{\text {noise }}$ denotes the amplitude of the noise-related non-fundamental harmonic with the highest amplitude, for the $k$-th excited frequency. This parameter can be obtained using the $\wp U$ method [39]. The following parameter can be defined:

$$
\Gamma_{k}=\sum_{\substack{i=2 \\ i \neq \max }}^{+\infty}\left(\Delta U_{k, i}^{\text {noise }}\right)^{2}=\chi_{k}^{2}-\left(\Delta U_{k, \max }^{\text {noise }}\right)^{2}
$$

Parameter $\Gamma_{k}$ clusters, for the $k$-th excited frequency, the information of all the noiserelated non-fundamental harmonics except the one with the highest amplitude. The relative comparison of parameter $\Gamma_{k}$ (i.e. all the noise components, except the most important one) and parameter $\Delta U_{k, \max }^{\text {noise }}$ (i.e. the most important noise component) is useful since it quantifies the relative importance of the most important noise component with respect to the rest of noise components. In this work, the following noise homogeneity coefficient was defined for the $k$-th excited frequency:

$$
\phi_{k}=\frac{\left(\Delta U_{k, \text { max }}^{\text {noise }}\right)^{2}}{\Gamma_{k}}=\frac{\left(\Delta U_{k, \text { max }}^{\text {noise }}\right)^{2}}{\chi_{k}^{2}-\left(\Delta U_{k, \text { max }}^{\text {noise }}\right)^{2}}
$$

The noise homogeneity coefficient, $\phi$, defined in this way quantifies the relative weight of the most important noise component with respect to the sum of the rest of the noise-related-components. A large value of $\phi_{k}$ suggests that for the $k$-th excited frequency, the most important noise-related-component has a big weight with respect to the rest of the noise-related-components (i.e. most important component domination); whereas, a small value of $\phi_{k}$ suggests that for the $k$-th excited frequency, the most important noise-related-component has a low weight with respect to the rest of the noise-related-components (i.e. rest of components domination).

In this work, the value of $\Delta U_{k, \text { max }}^{\text {noise }}$ for each excited frequency was obtained using the $\wp U$ method [39]. Then, using equation (11) with the corresponding $\chi$ values, parameter $\phi_{k}$ was calculated for each excited frequency. Figure 4 shows the obtained results. For most excited frequencies, the noise homogeneity coefficient has roughly the same value, of around 0.05 . This observation implies that for most of the excited frequencies, the most important noise-related-component represents approximately the same fraction of the noise-related-components. Since 0.05 is significantly lower than 1, it can be concluded that for most excited frequencies there is a domination of the rest of noise-related components over the most important noise-relatedcomponent. This means that a large amount of noise-related information is lost if the most important component is considered instead of the whole set of components. 
Four excited frequencies present significantly higher values of the noise homogeneity coefficient: $500 \mathrm{~Hz}, 1000 \mathrm{~Hz}, 2000 \mathrm{~Hz}$ and $4000 \mathrm{~Hz}$. This implies that for these 4 excited frequencies, the most important noise-related component represents a greater fraction of the noise-related components. For example, for an excited frequency of $1000 \mathrm{~Hz}, \phi_{k} \approx 5$ : for this excited frequency, the most important noise-relatedcomponent is far more significant than the sum of all the other components (i.e. most important component domination). However, this change of dominance only happens at two excited frequencies: $500 \mathrm{~Hz}$, and $1000 \mathrm{~Hz}$. These are the only 2 excited frequencies in this system, in which the most important component can be considered instead of the whole set of components without an appreciable loss of noise-related information.

With the analysis presented in this section, the noise structure of the system has been fully determined. From a qualitative analysis of the noise structure, the main noise source can be elucidated: in this case, the main noise source is the electric coupling of the system with the electric grid. From a quantitative analysis of the noise structure, the effective noise level for each excited frequency can be obtained. From this information, the frequencies that are more affected by noise (i.e. require higher perturbation amplitudes in order to overcome it) can be identified. Moreover, the noise structure allows to determine for which excited frequencies, the most important noise-related component can be considered alone, neglecting the rest of components; and for which excited frequencies, all the component have to be considered in order to avoid an appreciable loss of noise-related information.

\subsection{Nonlinear effects}

Since the optimum perturbation amplitude is defined by the tradeoff between SNR improvement and nonlinear effects, the next step after characterizing the noise structure is the quantification of the nonlinear effects displayed at each excited frequency for each perturbation amplitude. As stated in section 2 , the great advantage of the THD method is that it allows the simultaneous quantification of noise and nonlinear effects. Expression (8) was used here in order to quantify the nonlinear effects. The obtained results are shown in figure 5.

On the one hand, for low perturbation amplitudes (i.e. lower than $2 \mathrm{~mA}$ ), the nonlinear effects are roughly zero for all the excited frequencies. It can be deduced, that for these low amplitudes, the nonlinear effect generation is negligible at every excited frequency. On the other hand, for high perturbation amplitudes (i.e. higher or equal to $2 \mathrm{~mA}$ ), two clear zones can be identified on the $\mathcal{T H} \mathcal{H} U^{\text {nonlinear }}$ curves. For high frequencies, $\mathcal{T H} \mathcal{H} U^{\text {nonlinear }}$ is frequency independent and negligible for every perturbation amplitude; thus, for high frequencies, no significant nonlinear effects are 
generated even for very high perturbation amplitudes. On the contrary, for low frequencies, $\mathcal{T} \mathcal{H} \mathcal{D} U^{\text {nonlinear }}$ is clearly frequency dependent: $\mathcal{T} \mathcal{H} \mathcal{D} U^{\text {nonlinear }}$ increases when the excited frequency decreases. It can be deduced that, for a given perturbation amplitude, the highest nonlinear effects are generated for the minimum frequency at which the EIS spectrum was measured. Furthermore, for a given excited frequency, $\mathcal{T H \mathcal { H }} U^{\text {nonlinear }}$ increases with an increase of the perturbation amplitude. This implies that higher nonlinear effects are generated at higher perturbation amplitudes.

The threshold frequency concept arises from the fact that there is a difference of nonlinear effect generation between high and low frequencies: for frequencies above the threshold frequency, no significant nonlinear effects are generated, even for very high perturbation amplitudes. For this system, the threshold frequency is a bit above $10 \mathrm{~Hz}$. In EIS context, the concept of threshold frequency was introduced by Agarwal and co-workers [47]. This threshold frequency has been observed in several systems [60]. For systems that exhibit this threshold frequency, very high amplitudes can be applied at high frequencies (i.e. higher than the threshold frequency) without worrying about the generation of nonlinear effects.

\subsection{Constant amplitude strategy}

As presented in section 3, the selection of the perturbation amplitude for the constant amplitude strategy was done using the critical parameter curve, which consists in the representation of $\mathcal{T H} \mathcal{H} U_{c}$ versus $\Delta I$. The experimental critical curve obtained in this case is shown in figure 6 . In the aforementioned figure, for each perturbation amplitude, the critical frequency is indicated next to the corresponding point of the critical curve. The critical curve displays a two trend shape, already reported in previous works [39, 40,81]. In these works, a thorough discussion of the origin of the two trends has been presented. In short, the 2 trends arise from the change of domination of the phenomena that happen when the perturbation amplitude is increased: for low perturbation amplitudes, the SNR improvement dominates, and therefore the critical parameter decreases with the perturbation amplitude (i.e. linear behavior zone); whereas, for high perturbation amplitudes, the generation of nonlinear effects dominates, resulting in a critical parameter increase with the perturbation amplitude (i.e. nonlinear behavior zone). The point at which the trend reverses, marks the limit between the pseudo-linear behavior zone and the nonlinear behavior zone. These zones are identified on figure 6: the first one is colored with a green background, whereas the second one is colored with a blue background.

Moreover, a critical frequency shift is observed in the transition from the linear behavior zone to the nonlinear behavior zone. In the first zone, the critical frequency is 
$25 \mathrm{~Hz}$ for every perturbation amplitude. As it was deduced from the analysis of the noise structure in section $5.2,25 \mathrm{~Hz}$ is the excitation frequency that presents higher levels of effective noise in this system. And since the linear behavior zone is associated to SNR improvement domination, it is natural that the critical frequency in this zone corresponds with the frequency at which the effective noise level is maximum. In the second zone, the critical frequency is $5 \mathrm{mHz}$ (i.e. the minimum frequency at which the EIS spectra were measured) for every perturbation amplitude. As it was deduced from the analysis of the nonlinear effects in section $5.3,5 \mathrm{mHz}$ is the excitation frequency that presents higher levels of nonlinear effects in this system. And since the nonlinear behavior zone is associated to the domination of nonlinear effect generation, it is natural that the critical frequency in this zone corresponds with the frequency at which the nonlinear effects are maximum. This critical frequency shift is an indicator of the domination switch from noise to nonlinear effects [40]; and therefore, it marks as well the frontier between the pseudo-linear and the nonlinear behavior zones.

As stated in section 3, the optimum perturbation amplitude for a constant amplitude strategy, corresponds with the perturbation amplitude that separates the pseudolinear behavior zone from the nonlinear behavior zone; since this is the perturbation amplitude that maximizes the SNR, while maintaining the generation of nonlinear effects under a non-significant level. In practice, this is the same to say that the optimum perturbation amplitude is given by the horizontal coordinate of the minimum point of the critical parameter curve. Applying this argument to figure 6 , the optimum perturbation amplitude for a constant amplitude strategy for this system was obtained: it belongs to the range 1 to $3 \mathrm{~mA}$. Restricting the options to the perturbation amplitudes applied in this work, the best perturbation for EIS measurements in this system, using a constant amplitude strategy, is a $2 \mathrm{~mA}$ amplitude perturbation.

The optimum perturbation for a constant amplitude strategy obtained here, using the THD method, is exactly the same that the one that was obtained applying the $\wp U$ method to this system in a previous work [40]. This arises from the fact that in the constant amplitude strategy, the optimum amplitude is defined by the critical frequencies (i.e. the critical frequency of the linear behavior zone, and the one of the nonlinear behavior zone). For these frequencies, the information contained in the $\wp U$ parameter is equivalent to the information contained in THD. Thus, for the constant amplitude strategy, both methods give equivalent results.

\subsection{Frequency dependent amplitude strategy}

As presented in section 3, the selection of the perturbation amplitudes for the frequency dependent amplitude strategy was done using the individual frequency THD plots, which consists in the representation of $\mathcal{T H} \mathcal{H} U$ versus $\Delta I$ for each excited 
frequency. Figure shows the individual frequency THD plot of four excited frequencies: $10 \mathrm{kHz}, 25 \mathrm{~Hz}$ (i.e. critical frequency of the linear behavior zone), $5 \mathrm{~Hz}$ and $5 \mathrm{mHz}$ (i.e. critical frequency of the nonlinear behavior zone). For $10 \mathrm{kHz}$ and $25 \mathrm{~Hz}$, the individual frequency THD plots are one-trend-plots: for these frequencies, $\mathcal{T H} \mathcal{H} U$ decreases with $\Delta I$ for all the perturbation amplitudes. This implies that these frequencies only exhibit a linear behavior zone, and do not present a nonlinear behavior zone. In other words, for these excited frequencies, the system behaves pseudo-linearly for every perturbation amplitude. This observation is consistent with the results presented in section 5.3: both, $10 \mathrm{kHz}$ and $25 \mathrm{~Hz}$, are above the threshold frequency of the system, and therefore, no significant nonlinear effects are generated at these frequencies, even for very high perturbation amplitudes. This implies that the maximum perturbation amplitude (i.e. $10 \mathrm{~mA}$ ) can be used for these frequencies so that the SNR is maximized. On the contrary, for $5 \mathrm{~Hz}$ and $5 \mathrm{mHz}$, the individual frequency THD plots are two-trend-plots: for these frequencies, the individual frequency THD plots present the two trends that were already reported from the critical parameter curve in section 5.4. There, the origin of the 2 trends was discussed in detail. As stated in section 3 , the optimum amplitude for a given excited frequency can be obtained from the frontier between the linear behavior zone (i.e. green zone) and the nonlinear behavior zone (i.e. blue zone) of the individual frequency THD plot for that excited frequency: that is, from the minimum of the individual frequency THD plot. According to this argument, the optimum perturbation amplitudes for the 4 presented frequencies are: $10 \mathrm{~mA}$ for $10 \mathrm{kHz}$ and $25 \mathrm{~Hz}, 3 \mathrm{~mA}$ for $5 \mathrm{~Hz}$, and $1 \mathrm{~mA}$ for $5 \mathrm{mHz}$.

For the sake of succinctness, in this work the individual frequency THD plots of only 4 excited frequencies were presented. However, there is one for each frequency at which the EIS spectra were measured. Using the method described above with each one of the individual frequency THD plots, the optimum amplitude for each excited frequency was obtained. Figure 8.a shows the obtained results. The first observation that can be extracted from this figure is that the optimum perturbation amplitude is strongly frequency dependent in this system. Three zones can be identified. On the one hand, in zone III (i.e. high frequencies), the maximum perturbation amplitude (i.e. $10 \mathrm{~mA}$ ) should be used. In this frequency zone, frequencies are above the threshold frequency of the system; and as explained when the optimum amplitudes for $10 \mathrm{kHz}$ and $25 \mathrm{~Hz}$ were presented, for frequencies above the threshold frequency, the maximum amplitude should be used in order to maximize the SNR since no nonlinear effects are generated for these frequencies. On the other hand, in zone I (i.e. low frequencies), low perturbation amplitudes should be used. As it was observed in section 5.3, the frequencies in this zone are the frequencies that present higher nonlinear effects. So, even if some of these frequencies (especially the very low frequencies) present significant effective noise levels (see section 5.2), the high nonlinear effects generated at these frequencies cause the nonlinear effects to 
overcome the noise even for low perturbation amplitudes. Therefore, the optimum perturbation amplitude for these frequencies is low. Finally, zone II (i.e. intermediate frequencies) is a transition zone between zone I, where low perturbation amplitudes are required; and zone III, in which high perturbation amplitudes have to be used. This zone corresponds to zone $B$ identified on the noise structure in section 5.2: frequencies in this zone display higher effective noise levels. Moreover, for these frequencies, the generation of nonlinear effects increases when frequency decreases: for the highest frequency in zone II it is relatively low, while that for the lowest frequency in zone II it is relatively high. The combination of these two facts leads to the pattern observed in zone II of figure 8.a. Moreover, in zone II, two peaks can be identified: $25 \mathrm{~Hz}$ and $12.5 \mathrm{~Hz}$. As observed in section 5.2, these frequencies are the frequencies that present the highest levels of effective noise: for these excited frequencies, the effective noise level is so high that it masks the nonlinear effects even when the maximum perturbation amplitude is used. The result is that the optimum perturbation amplitude for these frequencies corresponds with the maximum amplitude (i.e. $10 \mathrm{~mA}$ ).

Additionally, the red horizontal line of figure 8.a indicates the optimum amplitude for the constant amplitude strategy, obtained in the previous subsection. On the one hand, in zone I, the constant-amplitude-strategy amplitude is slightly higher than the frequency-dependent-amplitude-strategy amplitudes. Thus, for the frequencies in zone I, the constant amplitude strategy may generate some nonlinear effects. On the other hand, in zone III, the constant-amplitude-strategy amplitude is significantly lower (i.e. $1 / 5$ ) than the frequency-dependent-amplitude-strategy amplitudes. This implies that for the frequencies in zone III, the constant amplitude strategy leads to a nonmaximum SNR. As it is natural, the optimum constant-amplitude-strategy amplitude is in between of the different frequency-dependent-amplitude-strategy amplitudes. However, it can be observed that it is much closer to the lower amplitudes (i.e. low frequencies) than to the higher amplitudes (i.e. high frequencies). This is positive, since nonlinear effects present more harmful effects on EIS measurement than nonoptimum SNR [18].

Figure 8.b shows the perturbation amplitude profile for a frequency dependent amplitude strategy, obtained using the $\rho U$ method in a previous work [40]. Comparing both subfigures of figure 8 , it can be observed that both, the THD method and the $\wp U$ method, give very similar results. This is quite natural, since they are both methods based on the analysis of the output signal in the frequency domain. However, in the $\wp U$ method, the transition zone (i.e. zone II) is greater at the expense of zone III. This is due to the fact that the THD method takes into account all the non-fundamental harmonics, whereas the $\rho U$ method only considers the most important nonfundamental harmonic. As it was observed in subsection 5.3 , in this system, a great 
amount of noise-related information is lost if the complete set of non-fundamental harmonics is replaced by the most important non-fundamental harmonic, except for two excited frequencies (i.e. $500 \mathrm{~Hz}$ and $1000 \mathrm{~Hz}$ ). It can be concluded that the results obtained using the $\rho U$ method are slightly biased due to the loss of noise-related information. For this reason, the THD method is somewhat superior to the $\rho U$ method. 


\section{$\underline{\text { 6. Conclusions }}$}

In conclusion, in this work the THD method has been successfully applied in order to select the optimum perturbation amplitude for EIS measurements in a particular model system, considering both, a constant amplitude strategy and a frequency dependent strategy. In addition to the optimum perturbation amplitude, the THD method also allows to obtain the noise structure. On the one hand, from a qualitative analysis of the noise structure, the main noise source can be elucidated. On the other hand, from a quantitative analysis of the noise structure, the effective noise level for each excited frequency can be obtained. Finally, the THD method also allows to quantify the generation of nonlinear effects at the different excited frequencies. From this information, the threshold frequency of the system can be obtained, in the case that the system presents one. All these arguments make the THD method a perfect method for the rigorous selection of the perturbation amplitude in EIS measurements.

The THD method is slightly superior to the $\wp U$ method (i.e. a method developed in earlier works, also based on the analysis of the output signal in the frequency domain), since it takes into account the information contained in all the non-fundamental components, whereas the $\wp U$ method only considers the most important nonfundamental component. Moreover, the THD method is easier to implement in practice, since there are already some EIS measurement commercial softwares available in the market (v.g. Zhaner ${ }^{\circledR}$ 's Thales ${ }^{\circledR}$ ) that are able to determine the total distortion values automatically during the measurement; while to the best of our knowledge, there is no commercial software able to calculate automatically parameter $\wp U$.

The amplitude optimization study presented in this work requires a morning of work to be done (its exact duration depends on the selected frequency range, number of frequencies and measurement parameters). It should be noted that this study has to be done only once for each new system, and not in each EIS measurement. Therefore, authors recommend to perform this amplitude optimization study as a preliminary study when starting to study a new system or when a modification is introduced in an already studied system (v.g. reference electrode change). And then, use the obtained optimum amplitude for all the subsequent EIS measurements performed for that system. 


\section{Nomenclature}

\section{Normal letters}

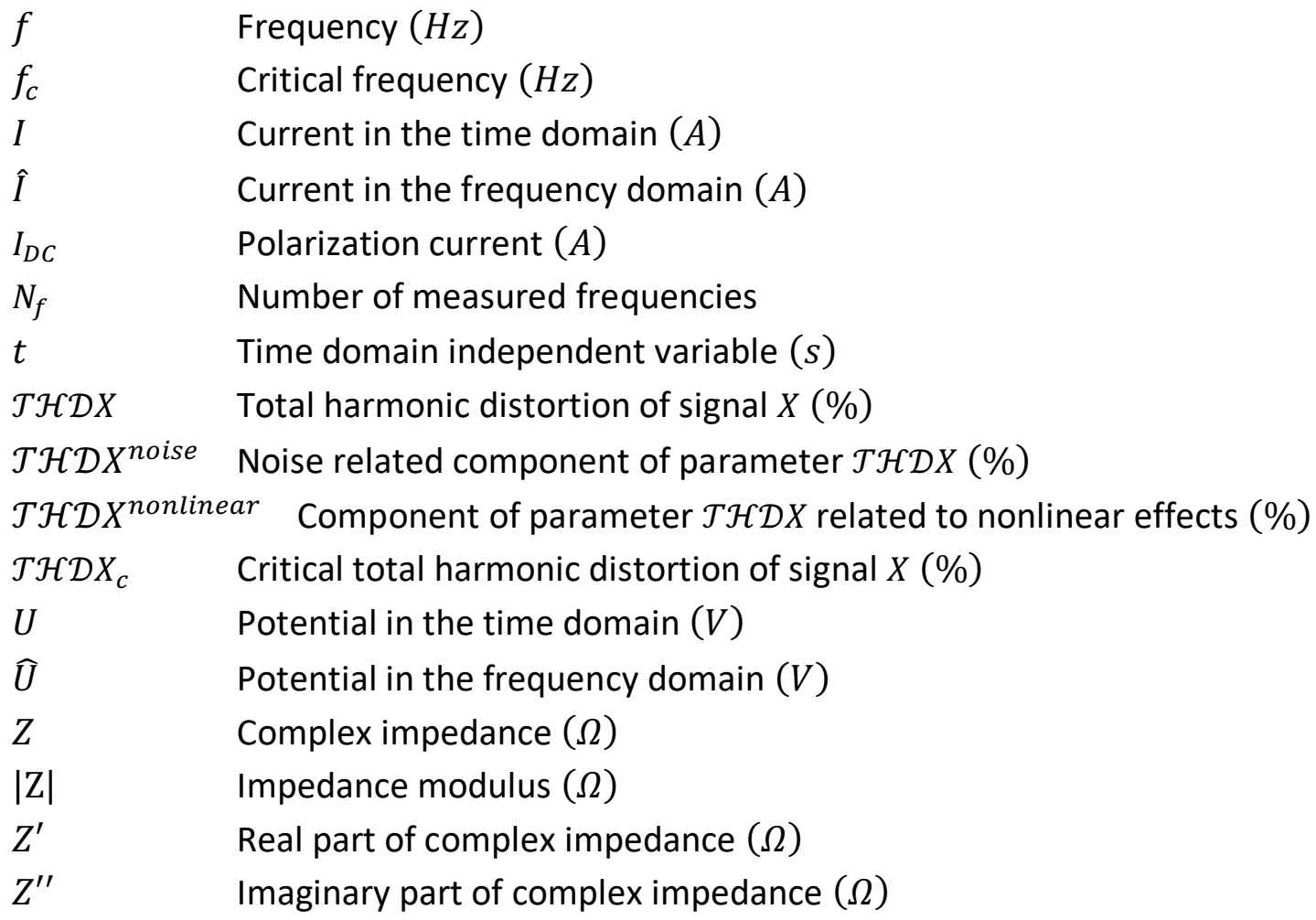

\section{Greek letters}

\begin{tabular}{|c|c|}
\hline$\Delta I$ & Galvanostatic perturbation amplitude $(A)$ \\
\hline$\Delta U_{i}^{\text {noise }}$ & $i$-th component of the output signal associated to noise $(V)$ \\
\hline$\Delta U_{k, \max }^{\text {noise }}$ & $\begin{array}{l}\text { Amplitude of the maximum noise-related non-fundamental harmonic, } \\
\text { for the } k \text {-th excited frequency }(V)\end{array}$ \\
\hline$\lambda$ & Effective noise parameter $(A)$ \\
\hline$\vartheta$ & Frequency domain independent variable $(\mathrm{Hz})$ \\
\hline$\phi$ & Noise homogeneity coefficient \\
\hline & Total noise parameter $(V)$ \\
\hline
\end{tabular}




\section{Acknowledgments}

The authors are very grateful to the Generalitat Valenciana for its economic support in form of Vali+d grant (Ref: ACIF-2013-268). 


\section{Bibliography}

[1] A. Sacco, Renew. Sust. Energ. Rev., 79, 814 (2017).

[2] J.R. Macdonald, Ann. Biomed. Eng., 20, 289 (1992).

[3] R. Yuksel, N. Uysal, A. Aydinli and H.E. Unalan, J. Electrochem. Soc., 165, A283 (2018).

[4] C. Wang, Y. Xiong, H. Wang, N. Yang, C. Jin and Qi. Sun, J. Electrochem. Soc., 165, A1054 (2018).

[5] X.H. Zhou, L. Cao, Z.H. Li, M.L. Zhang, W.M. Kang and B.W. Cheng, J. Electrochem. Soc., 165, A918 (2018).

[6] P.M. Ranjith, M.T. Rao, S. Sapra, I.I. Suni and R. Srinivasan, J. Electrochem. Soc., 165, C258 (2018).

[7] G. Ji, L. Fernández-Macía, B. Allaert, A. Hubin and H. Terryn, J. Electrochem. Soc., 165, C246 (2018).

[8] D. Horvath and M.F. Simpson, J. Electrochem. Soc., 165, C226 (2018).

[9] U. Bertocci, C. Gabrielli, F. Huet and M. Keddam, J. Electrochem. Soc., 144, 31 (1997).

[10] U. Bertocci, C. Gabrielli, F. Huet, M. Keddam and P. Rousseau, J. Electrochem. Soc., 144, 37 (1997).

[11] U. Bertocci and F. Huet, J. Electrochem. Soc., 144, 2786 (1997).

[12] E. Vijayakumar, S.H. Kang and K.S. Ahn, J. Electrochem. Soc., 165, F375 (2018).

[13] P.L. Kharel, F.P. Zamborini and B.W. Alphenaar, J. Electrochem. Soc., 165, H52 (2018).

[14] C. Gong, X. Hong, S. Xiang, Z. Wu, L. Sun, M. Ye and C. Lin, J. Electrochem. Soc., 165, H45 (2018).

[15] D. Mitra, P. Trinh, S. Malkhandi, M. Mecklenburg, S.M. Heald, M. Balasubramanian and S.R. Narayanan, J. Electrochem. Soc., 165, F392 (2018). 
[16] S. Yoon, J. Kim, J.H. Lim and B. Yoo, J. Electrochem. Soc., 165, H271 (2018).

[17] C.E. Frey, Q. Fang, D. Sebold, L. Blum and N.H. Menzler, J. Electrochem. Soc., 165, F357 (2018).

[18] J.J. Giner-Sanz, E.M. Ortega and V. Pérez-Herranz, Fuel Cells, 17, 391 (2017).

[19] N. Atar and M.L. Yola, J. Electrochem. Soc., 165, H255 (2018).

[20] K. Wippermann, J. Giffin and C. Korte, J. Electrochem. Soc., 165, H263 (2018).

[21] W.H. Zhou, H.H. Wang, W.T. Li, X.C. Guo, D.X. Kou, Z.J. Zhou, Y.N. Meng, Qi.W. Tian and S.X. Wu, J. Electrochem. Soc., 165, G3001 (2018).

[22] W. Nakpetpoon, T. Vongsetskul, P. Limthongkul and P. Tammawat, J. Electrochem. Soc., 165, A1140 (2018).

[23] J. Landesfeind, A. Eldiven and H.A. Gasteiger, J. Electrochem. Soc., 165, A1122 (2018).

[24] Q. Cheng and Y. Zhang, J. Electrochem. Soc., 165, A1104 (2018).

[25] S. Xia, F. Li, F. Cheng, X. Li, C. Sun, J.J. Liu and G. Hong, J. Electrochem. Soc., 165, A1019 (2018).

[26] Y. Garsany, R.W. Atkinson III, M.B. Sassin, R.M.E. Hjelm, B.D. Gould and K.E. Swider-Lyons, J. Electrochem. Soc., 165, F381 (2018).

[27] J.J. Giner-Sanz, E.M. Ortega and V. Pérez-Herranz, J. Power Sources, 379, 328 (2018).

[28] J.J. Giner-Sanz, E.M. Ortega and V. Pérez-Herranz, J. Power Sources, 381, 84 (2018).

[29] H. Liu, M.G. George, N. Ge, D. Muirhead, P. Shrestha, J. Lee, R. Banerjee, R. Zeis, M. Messerschmidt, J. Scholta, P. Krolla and A. Bazylak, J. Electrochem. Soc., 165, F3271 (2018).

[30] M.E. Orazem and B. Tribollet, Electrochemical Impedance Spectroscopy, John Wiley \& Sons, Hoboken (2008). 
[31] A. Lasia, Electrochemical Impedance Spectroscopy and its applications, Springer, London (2014).

[32] E. Barsoukov and J.R. Macdonald, Impedance Spectroscopy. Theory, experiment and applications, John Wiley \& Sons, New Jersey (2005).

[33] J.H. Choi, J.S. Park and S.H. Moon, J. Colloid Interf. Sci., 251, 311 (2002).

[34] D.D. Macdonald and E. Sikora, Electrochim. Acta, 43, 87 (1997).

[35] J.J. Giner-Sanz, E.M. Ortega and V. Pérez-Herranz, Int. J. Hydrogen Energ., 39, 13206 (2014).

[36] J.J. Giner-Sanz, E.M. Ortega and V. Pérez-Herranz, Fuel Cells, 15, 479 (2015).

[37] B. Hirschorn, B. Tribollet and M.E. Orazem, Isr. J. Chem., 48, 133 (2008).

[38] S.N. Victoria and S. Ramanathan, Electrochim. Acta, 56, 2606 (2011).

[39] J.J. Giner-Sanz, E.M. Ortega and V. Pérez-Herranz, Electrochim. Acta, 211, 1076 (2016).

[40] J.J. Giner-Sanz, E.M. Ortega and V. Pérez-Herranz, J. Electrochem. Soc., 164, H918 (2017).

[41] J.J. Giner-Sanz, E.M. Ortega and V. Pérez-Herranz, Fuel Cells, 16, 469 (2016).

[42] P. Gode, F. Jaouen, G. Lindbergh, A. Lundblad and G. Sundholm, Electrochim. Acta 48, 4175 (2003).

[43] X. Yuan, J.C. Sun, H. Wang and J. Zhang, J. Power Sources, 161, 929 (2006).

[44] Y. Fernández Pulido, C. Blanco, D. Anseán, V.M. García, F. Ferrero and M. Valledor, Measurement, 106, 1 (2017).

[45] F. Fasmin and R. Srinivasan, J. Solid State Electr., 19, 1833 (2015).

[46] J.J. Giner-Sanz, E.M. Ortega and V. Pérez-Herranz, Int. J. Hydrogen Energ., 40, 11279 (2015). 
[47] P. Agarwal, M.E. Orazem and L.H. Garcia-Rubio, J. Electrochem. Soc., 142, 4159 (1995).

[48] B.A. Boukamp, J. Electrochem. Soc., 142, 1885 (1995).

[49] B.A. Boukamp and J.R. Macdonald, Soild State Ionics, 174, 85 (1994).

[50] M.E. Orazem and B. Tribollet, Electrochim. Acta, 53, 7360 (2008).

[51] P.K. Shukla, M.E. Orazem and O.D. Crisalle, Electrochim. Acta, 49, 2881 (2004).

[52] M.E. Orazem, J. Electroanal. Chem., 572, 317 (2004).

[53] M.E. Orazem, P. Shukla and M.A. Membrino, Electrochim. Acta, 47, 2027 (2002).

[54] P. Agarwal, M.E. Orazem and L.H. Garcia-Rubio, Electrochim. Acta, 41, 1017 (1996).

[55] M.E. Orazem, P. Agarwal, C. Deslouis and B. Tribollet, J. Electrochem. Soc., 143, 948 (1996).

[56] P. Agarwal, O.D. Crisalle, M.E. Orazem and L.H. Garcia-Rubio, J. Electrochem. Soc., 142, 4149 (1995).

[57] P. Agarwal, M.E. Orazem and L.H. Garcia-Rubio, J. Electrochem. Soc., 139, 1917 (1992).

[58] M.E. Orazem, J.M. Esteban and O.C. Moghissi, Corrosion, 47, 248 (1991).

[59] M. Urquidi-Macdonald, S. Real and D.D. Macdonald, Electrochim. Acta, 35, 1559 (1990).

[60] B.Hirschorn and M.E. Orazem, J. Electrochem. Soc., 156, 345 (2009).

[61] J.J. Giner-Sanz, E.M. Ortega and V. Pérez-Herranz, Electrochim. Acta, 209, 254 (2016).

[62] W. Lai, Electrochim. Acta, 55, 5511 (2010).

[63] C. Montella and J.P. Diard, Nonlinear Impedance of Tafelian Electrochemical Systems, Wolfram Demonstrations Project, 2014, 
http://demonstrations.wolfram.com/NonlinearlmpedanceOfTafelianElectrochemicalSy stems/.

[64] C. Montella, J. Electroanal. Chem., 672, 17 (2012).

[65] J.P. Diard, B. Le Gorrec and C. Montella, Electrochim. Acta, 42, 1503 (1997).

[66] J.P. Diard, B. Le Gorrec and C. Montella, J. Electroanal. Chem., 432, 27 (1997).

[67] J.P. Diard, B. Le Gorrec and C. Montella, J. Electroanal. Chem., 432, 41 (1997).

[68] J.P. Diard, B. Le Gorrec and C. Montella, J. Electroanal. Chem., 432, 53 (1997).

[69] J.P. Diard, B. Le Gorrec and C. Montella, Electrochim. Acta, 39, 539 (1994).

[70] J.P. Diard, B. Le Gorrec and C. Montella, J. Electroanal. Chem., 377, 61 (1994).

[71] J. Smulko and K. Darowicki, J. Electroanal. Chem., 545, 59 (2003).

[72] K. Darowichi, Electrochim. Acta, 42, 1781 (1997).

[73] K. Darowichi, Electrochim. Acta, 40, 439 (1995).

[74] K. Darowichi, J. Electroanal. Chem., 394, 81 (1995).

[75] E. Van Gheem, R. Pintelon, J. Vereecken, J. Schoukens, A. Hubin, P. Verboven and O. Blajiev, Electrochim. Acta, 51, 1443 (2006).

[76] E. Van Gheem, R. Pintelon, J. Vereecken, J. Schoukens, A. Hubin, P. Verboven and O. Blajiev, Electrochim. Acta, 49, 4753 (2004).

[77] R. Pintelon, E. Louarroudi and J. Lataire, Automatica, 51, 308 (2015).

[78] R. Pintelon, E. Louarroudi and J. Lataire, IEEE T. Instrum. Meas., 62, 3361 (2013).

[79] G.S. Popkirov and R.N. Schindler, Electrochim. Acta, 40, 2511 (1995).

[80] G.S. Popkirov and R.N. Schindler, Rev. Sci. Instrum., 64, 3111 (1993).

[81] J.J. Giner-Sanz, E.M. Ortega and V. Pérez-Herranz, Electrochim. Acta, 186, 598 (2015). 
[82] D. Shmilovitz, IEEE T. Power Deliver., 20, 526 (2005).

[83] Q. Mao and U. Krewer, Electrochim. Acta, 103, 188 (2013).

[84] Q. Mao and U. Krewer, Electrochim. Acta, 68, 60 (2012).

[85] Q. Mao, U. Krewer and R. Hanke-Rauschenbach, Electrochem. Commun., 12, 1517 (2010).

[86] S. Thomas, S.C. Lee, A.K. Sahu and S. Park, Int. J. Hydrogen Energ., 39, 4558 (2014).

[87] J. Garcia-Antón, A. Igual-Muñoz, J.L. Guiñon, V. Pérez-Herranz, I. Herraiz-Cardona and E.M. Ortega, Horizontal cell for electro-optical analysis of electrochemical processes, ES patent P-2000002526, October 2000.

[88] I. Herraiz-Cardona, Desarrollo de nuevos materiales de electrodo para la obtención de hidrógeno a partir de la electrolisis alcalina del agua, PhD Tesis, Valencia, Universitat Politècnica de València (2012).

[89] J.J. Giner-Sanz, E.M. Ortega and V. Pérez-Herranz, Electrochim. Acta, 174, 1290 (2015). 


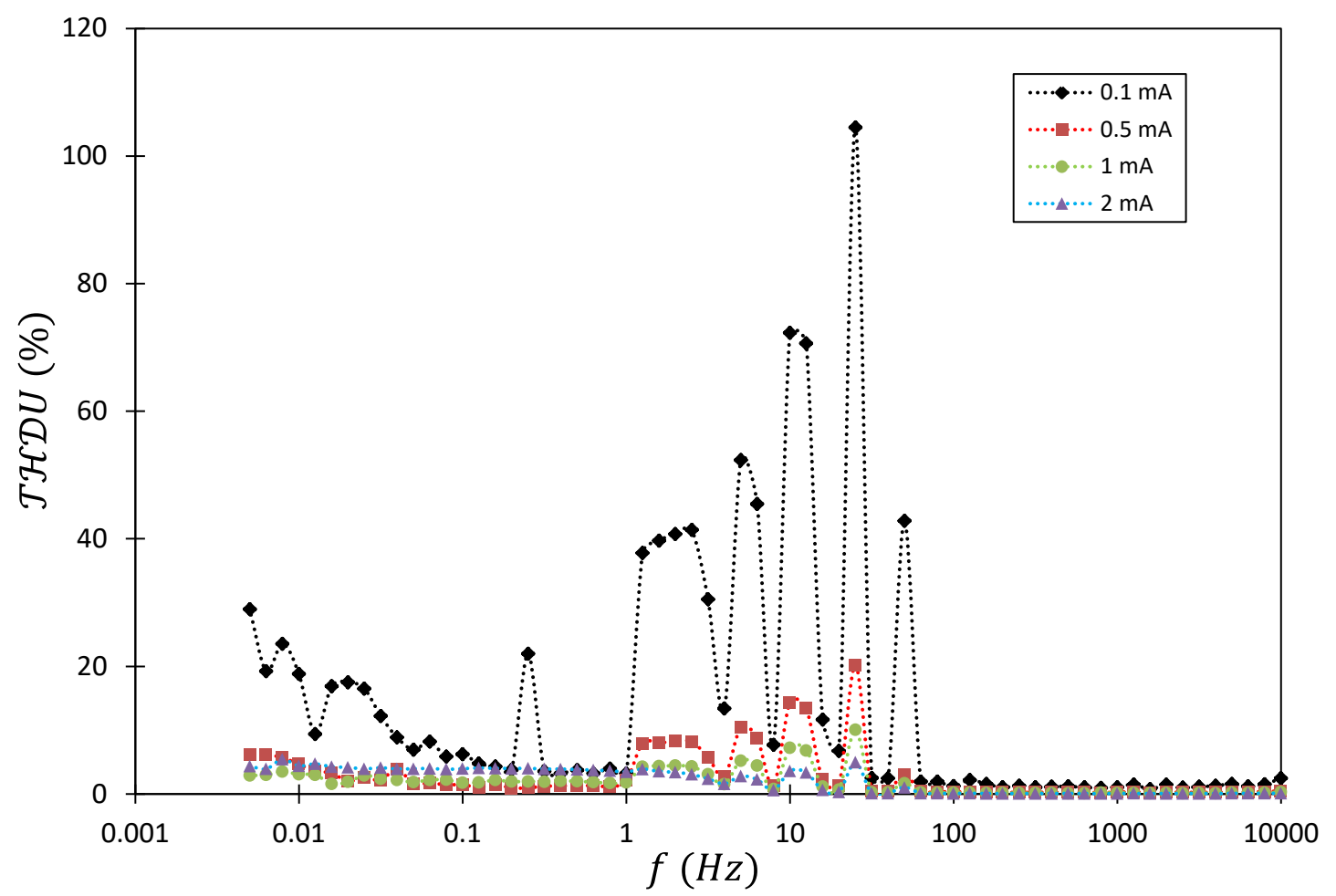

a.) Low perturbation amplitudes

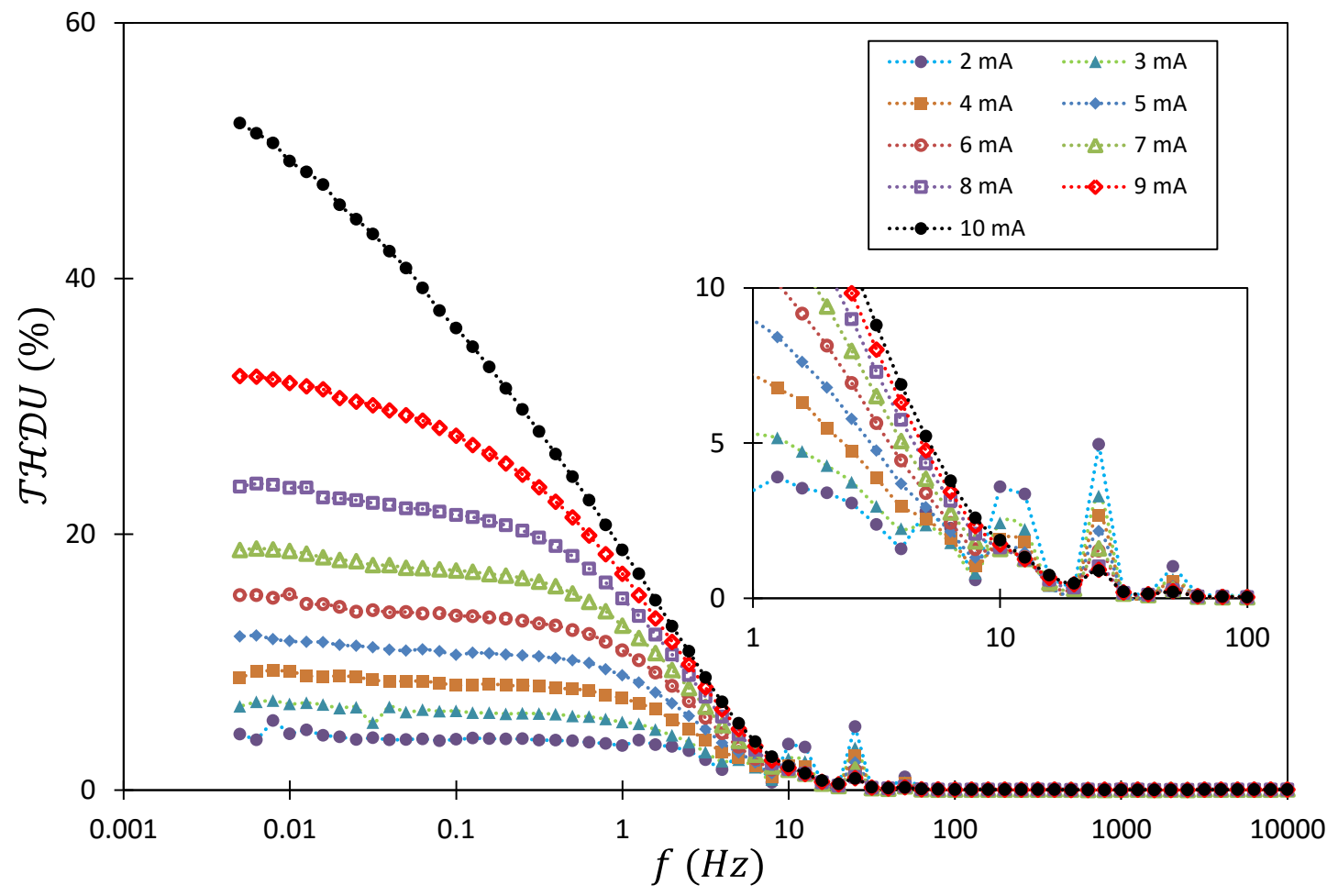

b.) High perturbation amplitudes

Figure 1. Output signal THD curves [81] 


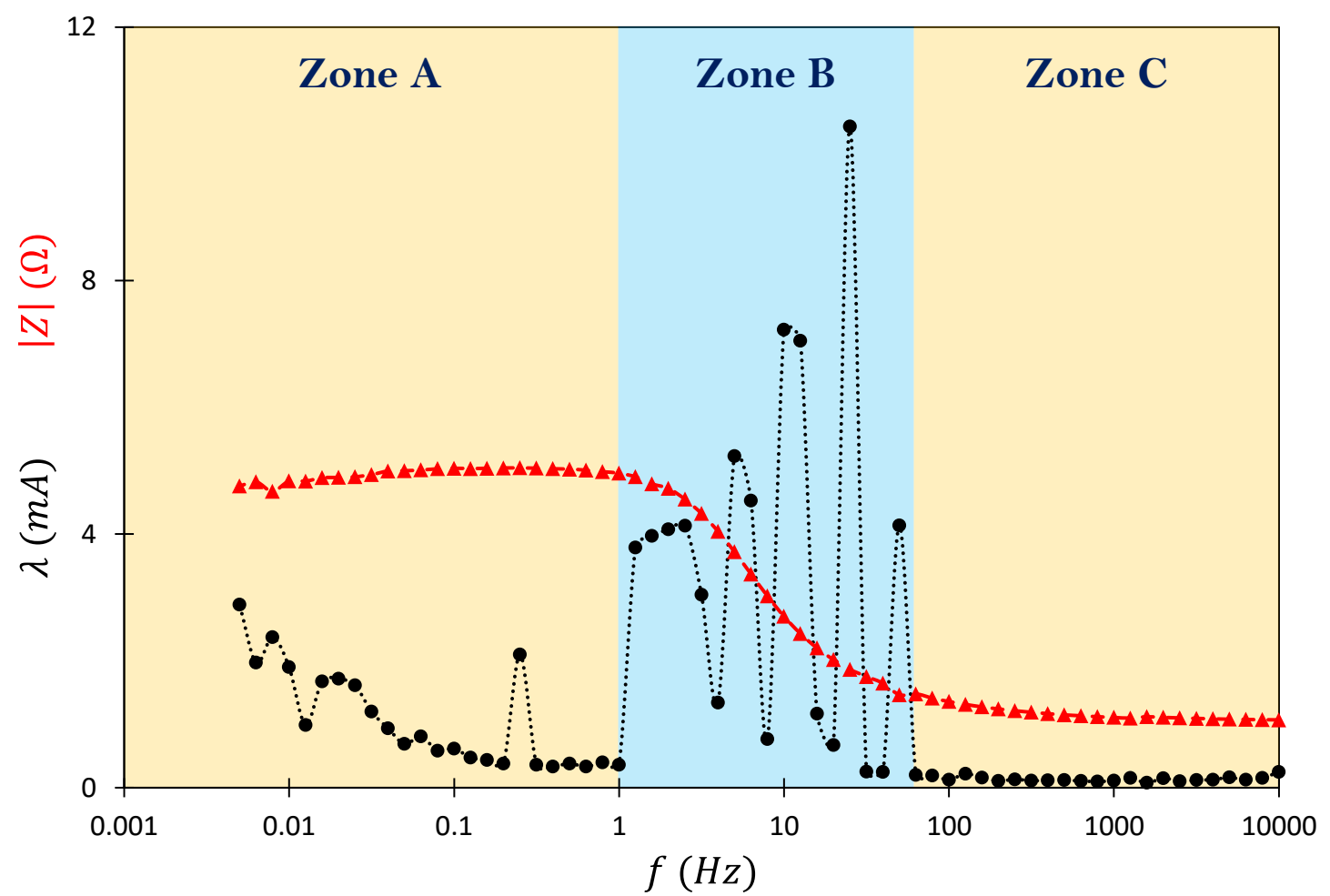

Figure 2. Effective noise parameter (black dots) and impedance modulus (red triangles) for each excited frequency 


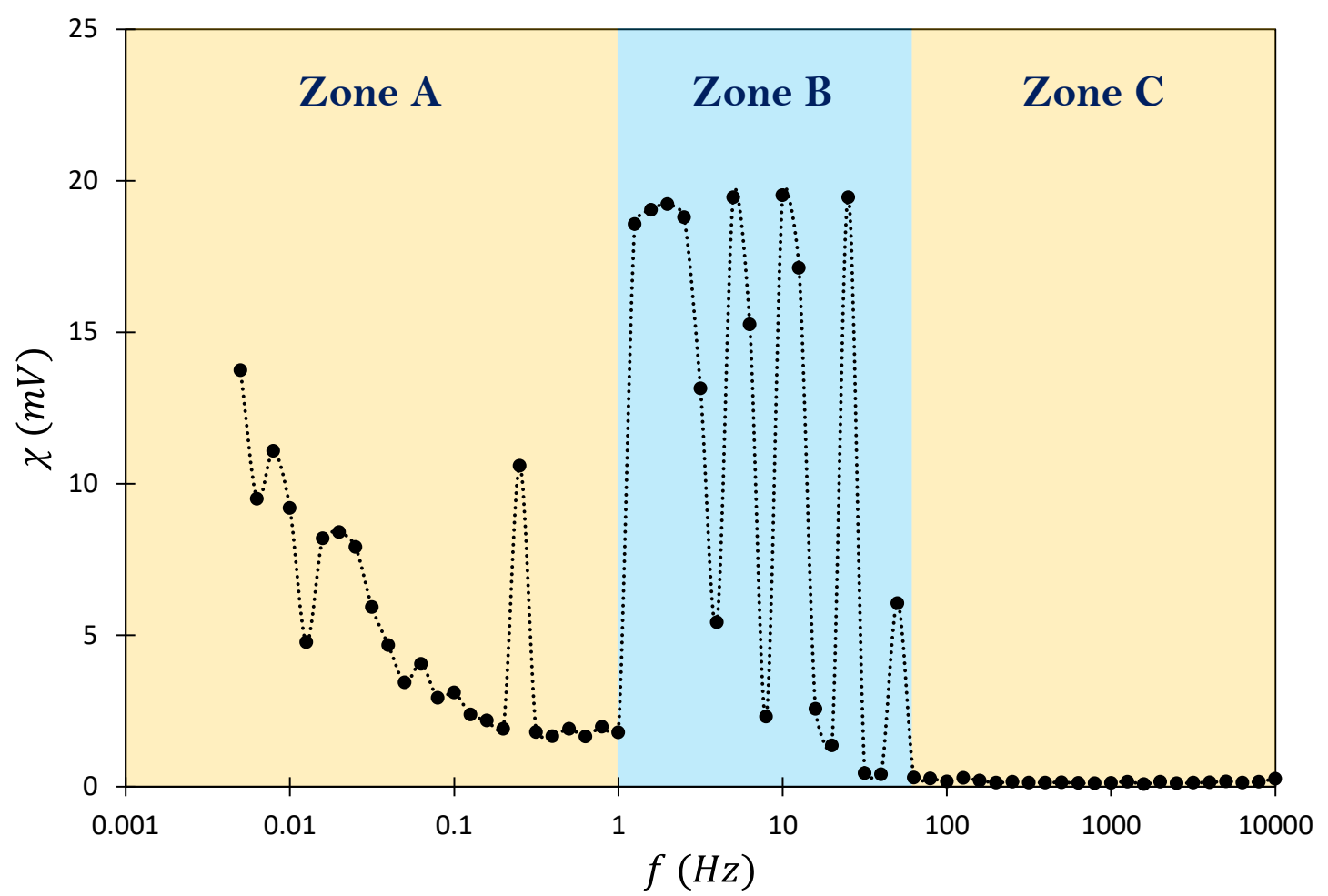

Figure 3. Total noise parameter for each excited frequency 


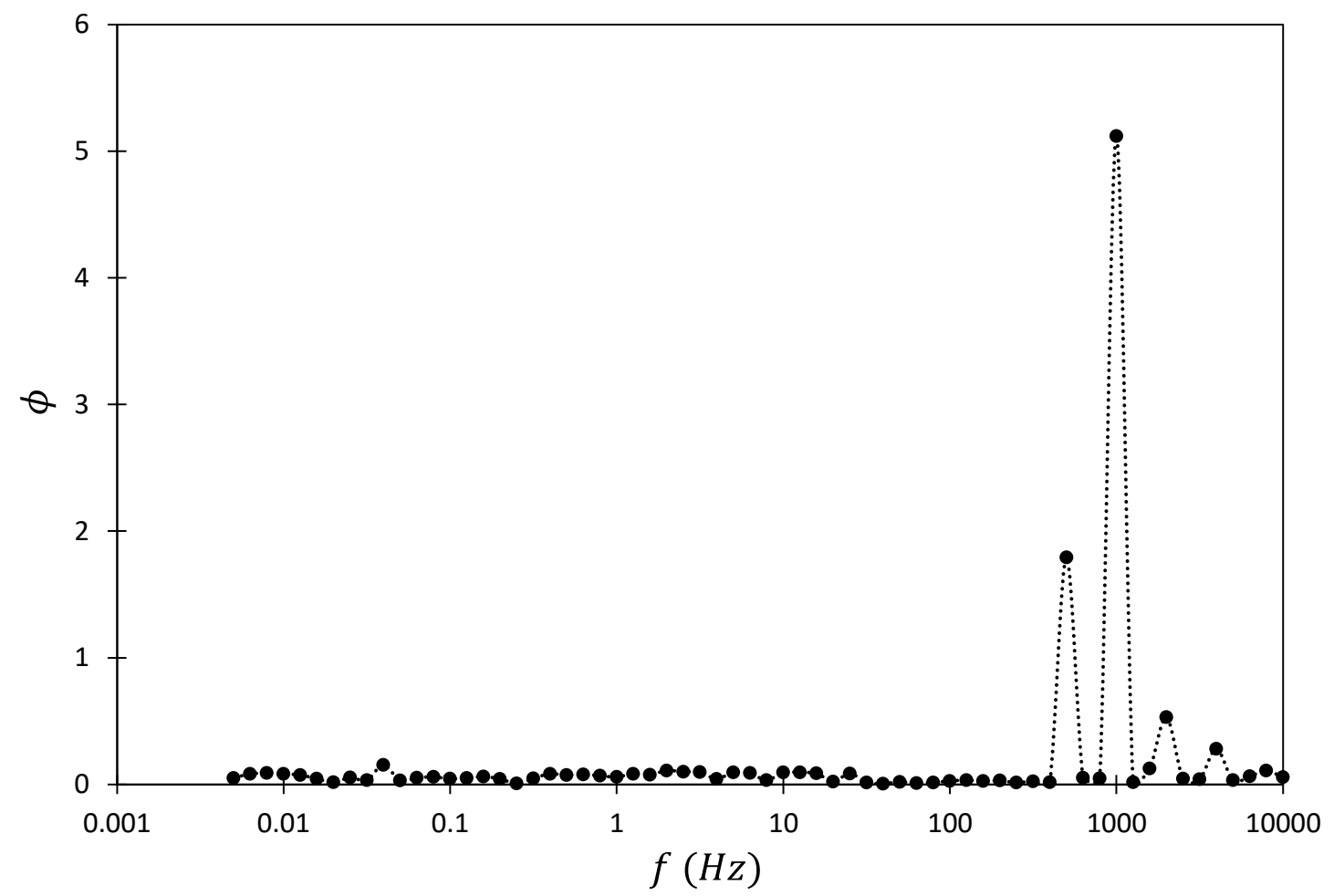

Figure 4. Noise homogeneity coefficient for each excited frequency 


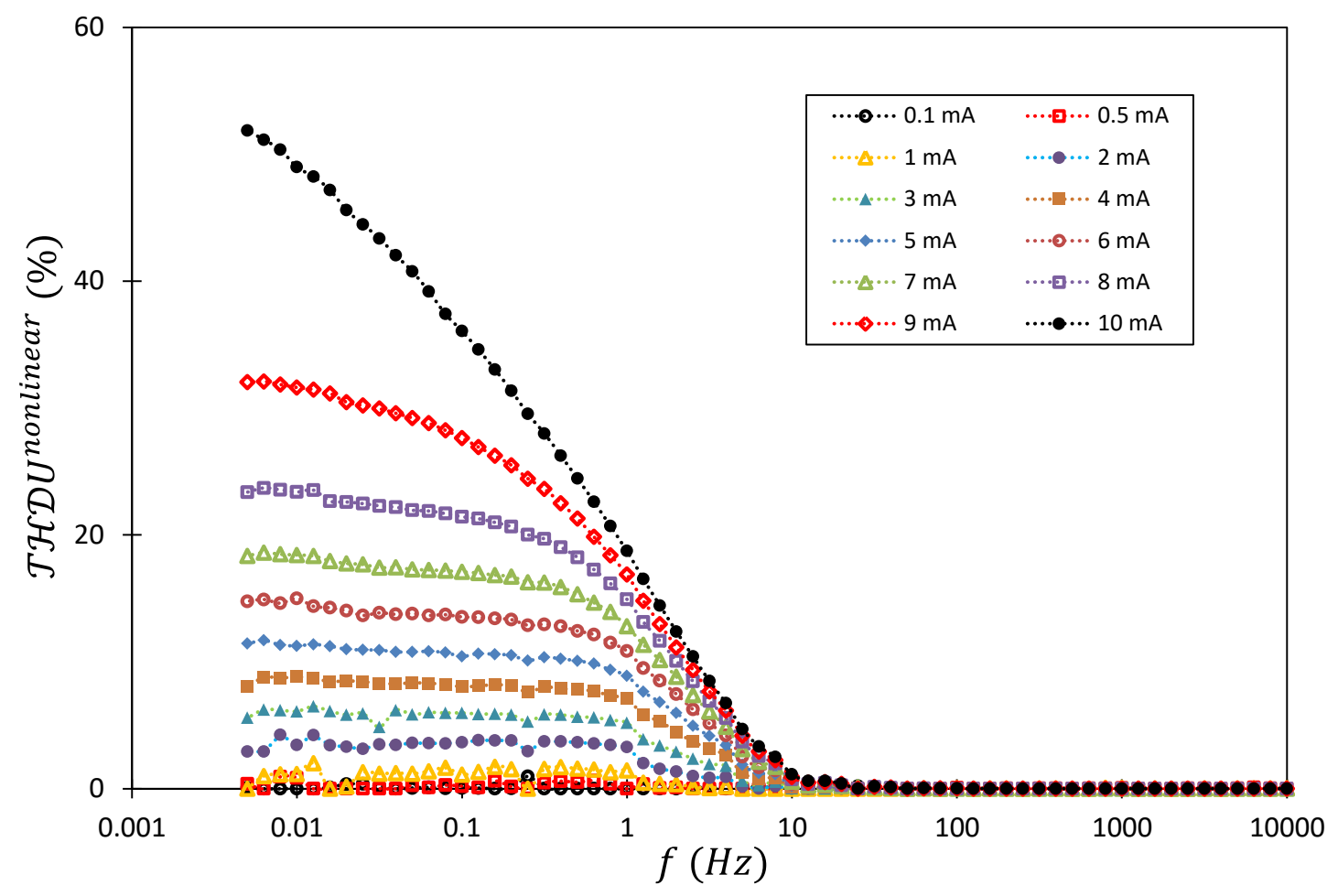

Figure 5. $\mathcal{T H} \mathcal{H} \mathcal{D}$ component related to nonlinear effects, for each excited frequency 


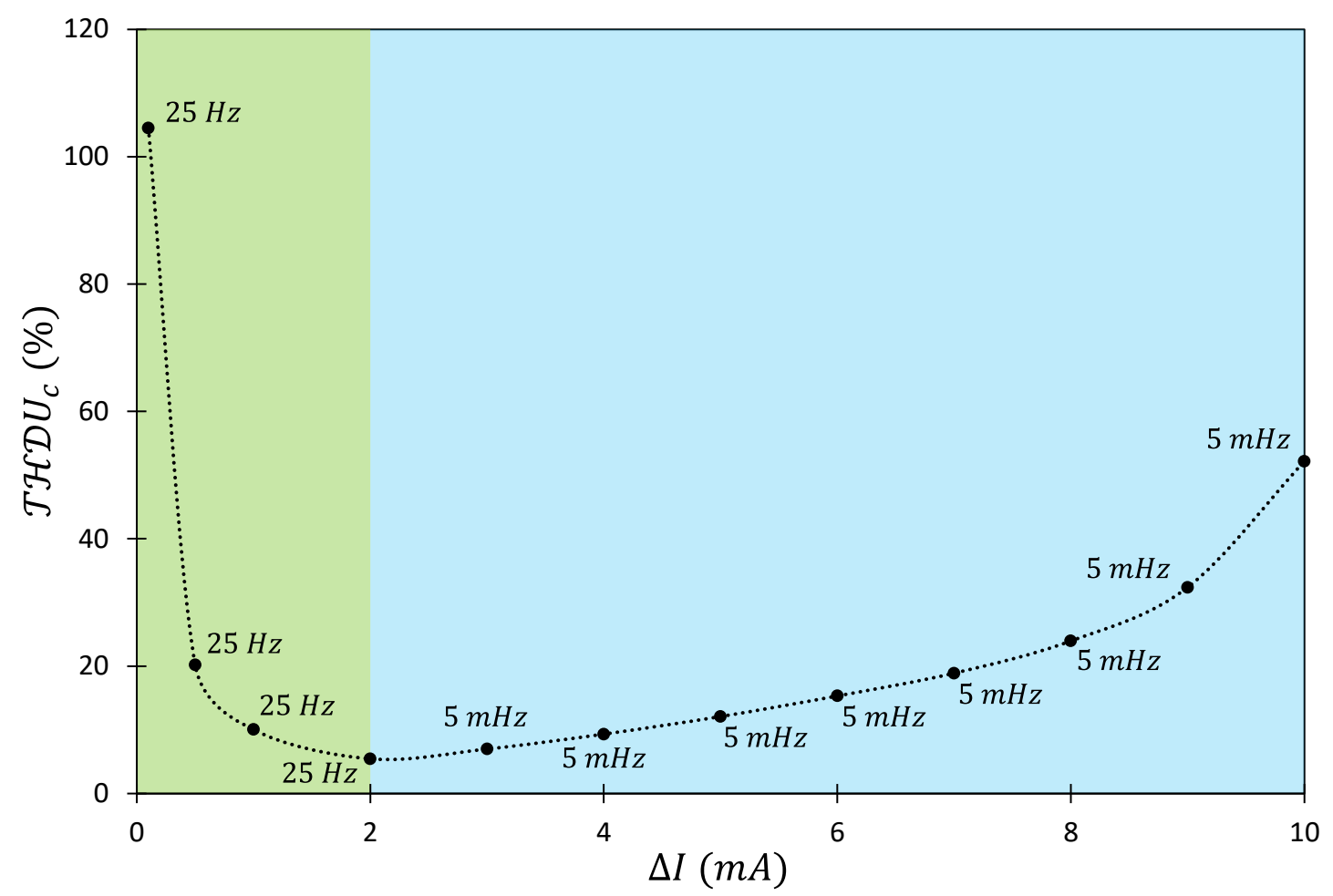

Figure 6. Critical parameter curve of the output signal 


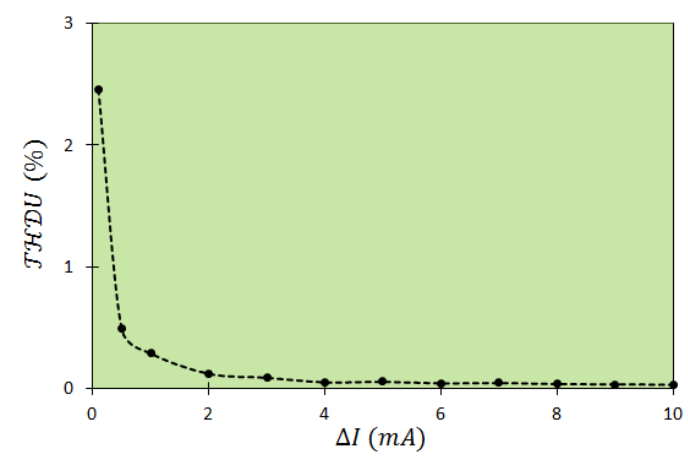

a.) $f=10 \mathrm{kHz}$

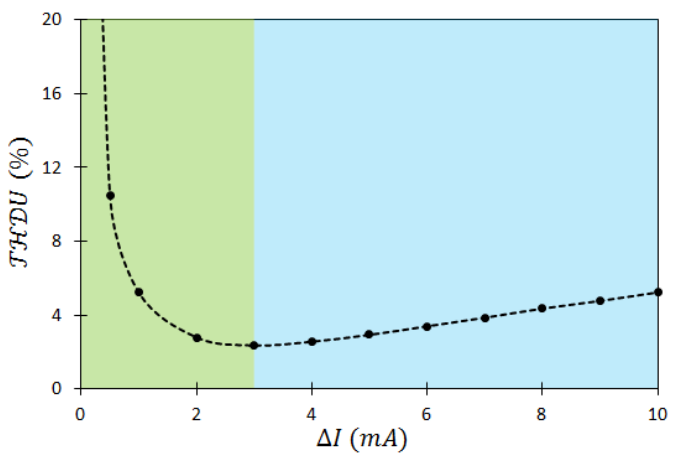

c.) $f=5 \mathrm{~Hz}$

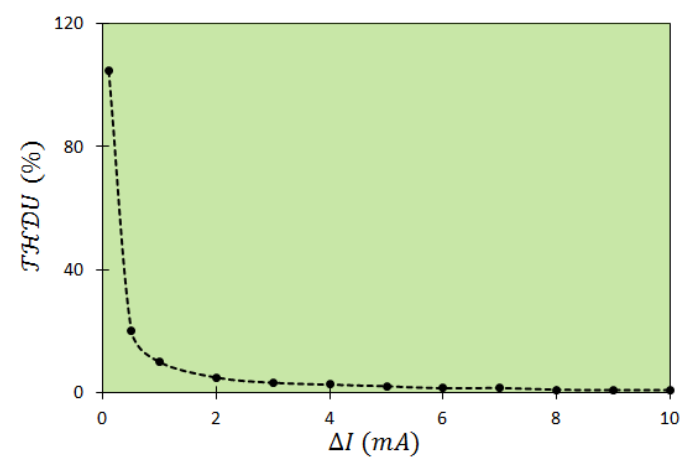

b.) $f=25 \mathrm{~Hz}$

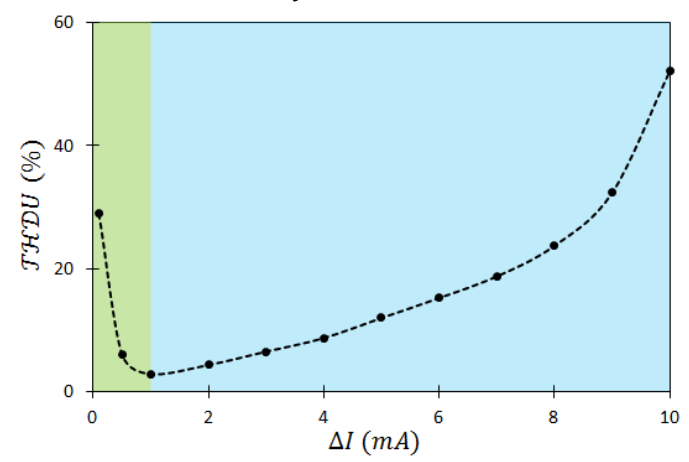

d.) $f=5 \mathrm{mHz}$

Figure 7. Individual frequency THD plots for different excitation frequencies 


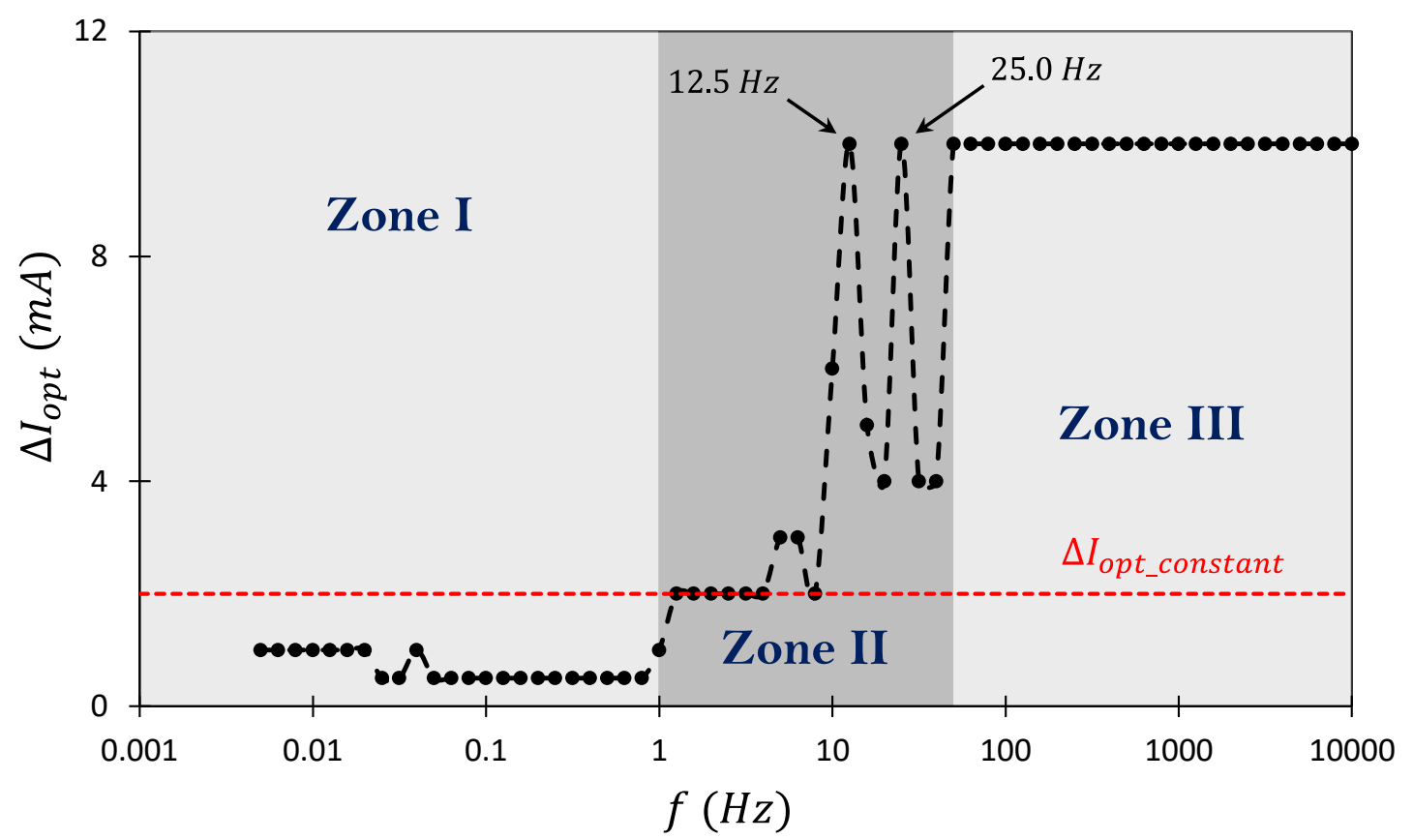

a.) Total harmonic distortion method (This work)

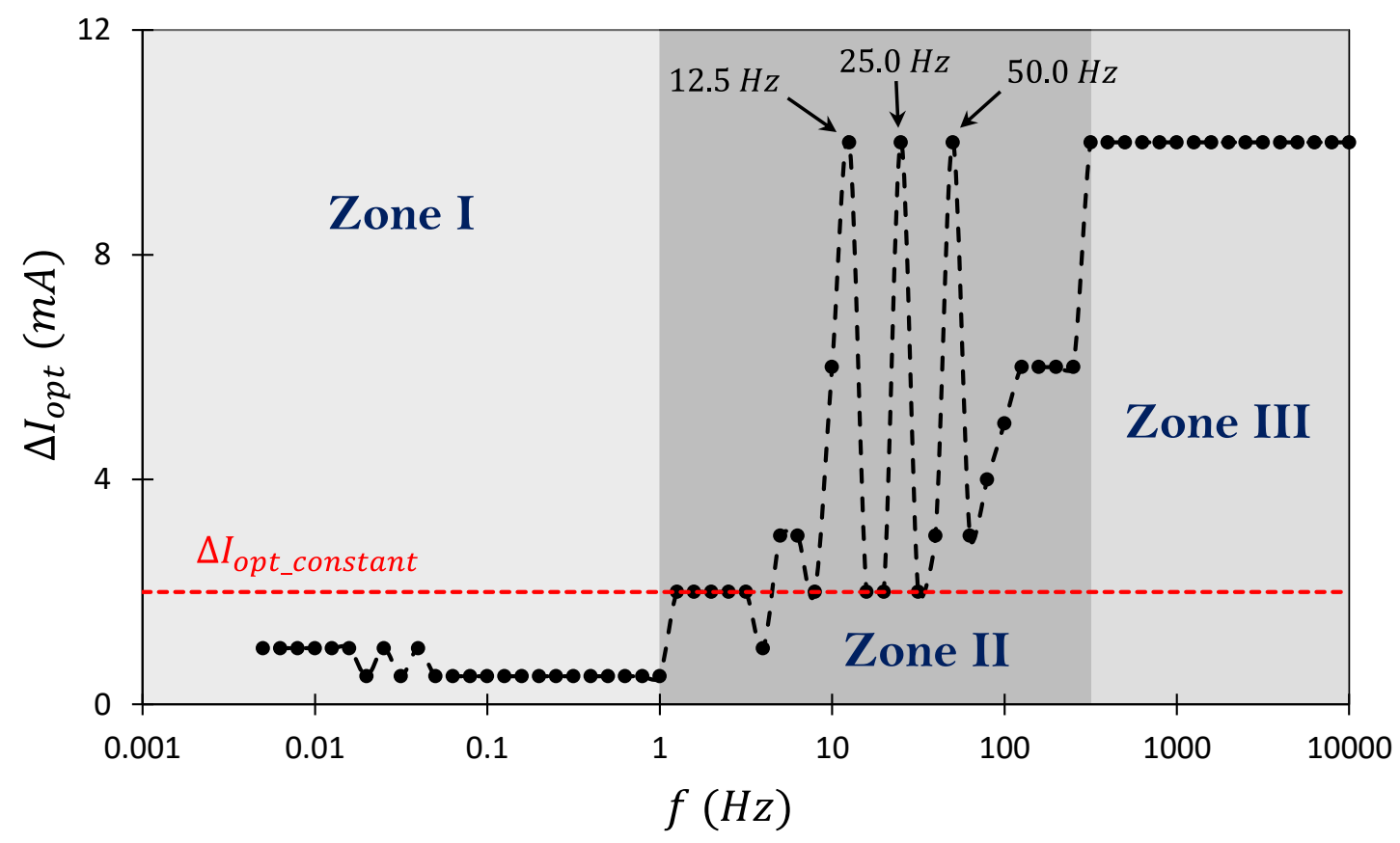

b.) $\wp U$ method ([40])

Figure 8. Optimum frequency dependent perturbation amplitude 